\title{
Vibrational self-alignment of a rigid object exploiting friction
}

\author{
B.G.B. Hunnekens $\cdot$ R.H.B. Fey $\cdot$ A. Shukla • \\ H. Nijmeijer
}

Received: 7 April 2010 / Accepted: 22 October 2010 / Published online: 6 November 2010

(C) The Author(s) 2010. This article is published with open access at Springerlink.com

\begin{abstract}
In this paper, one-dimensional self-alignment of a rigid object via stick-slip vibrations is studied. The object is situated on a table, which has a prescribed periodic motion. Friction is exploited as the mechanism to move the object in a desired direction and to stop and self-align the mass at a desired end position with the smallest possible positioning error. In the modeling and analysis of the system, theory of discontinuous dynamical systems is used. Analytic solutions can be derived for a model based on Coulomb friction and an intuitively chosen table acceleration profile, which allows for a classification of different possible types of motion. Local stability and convergence is proven for the solutions of the system, if a constant Coulomb friction coefficient is used. Next, near the desired end position, the Coulomb friction coefficient is increased (e.g. by changing the roughness of the table surface) in order to stop the object. In the transition region from low friction to high friction coefficient, it is shown that, under certain conditions, accumulation of the object to a unique end position
\end{abstract}

B.G.B. Hunnekens · R.H.B. Fey $(\varangle) \cdot$ H. Nijmeijer Department of Mechanical Engineering, Eindhoven University of Technology, P.O. Box 513, 5600 MB Eindhoven, The Netherlands

e-mail: R.H.B.Fey@tue.nl

\section{A. Shukla}

Department of Mechanical and Manufacturing Engineering, Miami University, EGB 56 Oxford, OH 45056, USA occurs. This behavior can be studied analytically and a mapping is given for subsequent stick positions.

Keywords Self-alignment · Friction · Stick-slip vibrations $\cdot$ Accumulation position $\cdot$ Positioning

\section{Introduction}

Accurate positioning of objects is very important in industrial applications, e.g. in printer heads, CD-players, pick-and-place machines, welding robots, etc. Usually, the positioning and tracking problem is tackled using closed-loop control using feedback to asymptotically stabilize the error dynamics. In this paper, the self-aligning positioning problem will be addressed. A rigid object is placed on a table which will be submitted to a periodic motion profile. Friction-based stick-slip vibrations will be used as a mechanism by which the rigid object (in this paper also referred to as: the mass) will move in a desired direction. By locally increasing the friction the mass will self-align at a desired end position.

Some (simplified) friction models for dynamics and control applications have been studied e.g. in [1]. An extensive treatment of friction modeling can be found in [2]. In literature, the dry-friction oscillator with Coulomb friction has received much attention. In this system, a mass experiencing Coulomb friction is connected to the world by a spring and a viscous damper, while an external periodic force acts on the mass, see 
[6, 13], and [21]. As early as 1930, Den Hartog gave the exact solution of a single degree of freedom harmonic oscillator with dry friction [6]. Cases where the static and dynamical friction coefficients are not equal to each other have been studied in [20]. Also, describing function approaches have been used to analyze the behavior of this type of system, see e.g. [7]. In [8], the method of averaging is used to analyze high-frequency vibration-induced movements of a mass between two layers of different friction, while in [23] the method of direct separation of motion (see [3]) is used to calculate equilibrium speeds (including zero speed) of a mass on a table vibrating at high frequency. In [24], analytical approximations for stick-slip vibration amplitudes are derived for the classical mass-on-movingbelt system. For the same system, in [10], bifurcations are studied, which are non-standard due to the discontinuous behavior of the dry friction force. In [5], the bifurcations associated with the appearance of stickslip vibrations are studied using a state variable friction law for a mass on a fixed horizontal surface, moving under the influence of a horizontal force transmitted by a linear spring whose other end is moving with constant velocity. A method of calculating exact analytic solutions for parts of the oscillation cycles, which are 'stitched' together, has been used in [4]; vibration-induced motions of a mass on a friction plane are studied, and optimal parameter values, for which maximum mean velocity is reached, have been determined.

Self-alignment of microparts is studied in [19], where the self-alignment is achieved using liquid surface tension. In [9], resonant magnetic microagents are used to move micro-robots using magnetic fields and vision-feedback for positioning applications. Modeling and closed-loop control of a 2-DOF micro-positioning device, using stick-slip motion based on piezoelectric materials, is treated in [17]. The work in [18] studies the use of a planar manipulator to position multiple objects on a vibrating surface using the frictional forces along with feedback control.

An important motivation for using self-alignment compared to conventional closed-loop control is to reduce cost by eliminating the need for feedback control equipment. Possible practical applications could include parts feeding, automated assembly, or inspection of parts. To the best knowledge of the authors, there is no literature available in which friction is exploited to move a mass in a desired direction and in which a mass self-aligns using friction, which motivates this study.

The paper is structured as follows. In Sect. 2, the two major objectives of this paper will be discussed. The focus in Sect. 3 will be on the dynamical model. The stopping regime will be discussed in detail. The table trajectory design will be studied in Sect. 4. In Sect. 5, the classification of different types of motion will be introduced. Local stability and convergence of solutions will be proven in Sect. 6. The positioning accuracy obtained, when the mass stops, will be investigated in Sect. 7. Finally, in Sect. 8, conclusions and some recommendations for future work will be given.

\section{Problem formulation}

The basic objective of this paper is to design a selfpositioning method, in which friction is used to move a mass on a table in a desired direction, using dry friction and periodic motion of the table. For the table, a suitable periodic motion profile must be designed that achieves this as fast as possible, given some actuator constraints. Furthermore, the mass needs to be positioned/stopped at a desired end position accurately. Summarizing, the two main objectives of this research are:

- Design a periodic table displacement signal $\tilde{y}(\tilde{t})$ such that the mass travels to a desired end location $\tilde{x}_{d}$ in the smallest possible process time $\tilde{T}_{p}$, where $\tilde{T}_{p}=\min (\tilde{W})$ and $\tilde{W} \in\left\{\tilde{t} \geq 0 \mid \tilde{x}(\tilde{t})=\tilde{x}_{d}\right\}$.

- Minimize the positioning error $\left|\Delta \tilde{x}_{d}\right|$ of the end position of the mass; in other words, minimize the interval $\left[\tilde{x}_{d}-\Delta \tilde{x}_{d}, \tilde{x}_{d}+\Delta \tilde{x}_{d}\right]$, in which the mass stops.

In this paper, the $\sim$ above a quantity means that the quantity is in SI units, whereas for dimensionless quantities the $\sim$ will be omitted.

\section{Modeling the system dynamics}

\subsection{Dynamical model}

Consider the system depicted in Fig. 1. For a practical application, the table displacement signal $\tilde{y}(\tilde{t})$ will have a zero average displacement. Therefore, it is assumed that $\tilde{y}(\tilde{t})$ will be periodic. The position of the 
Fig. 1 Schematic of the system

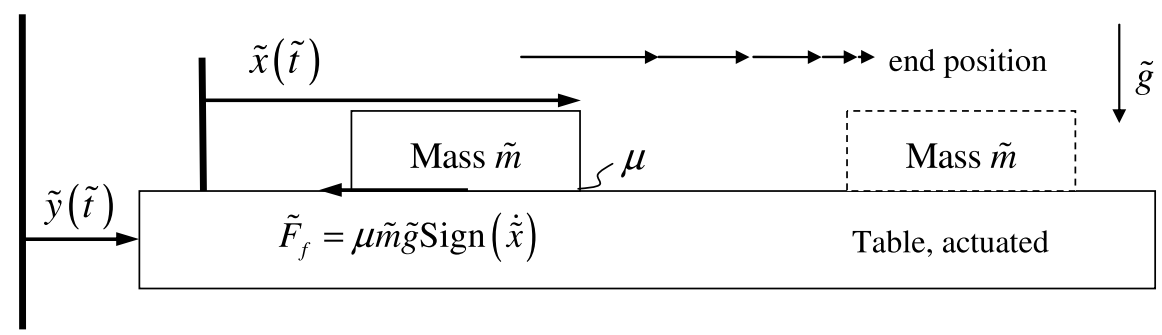

mass relative to the table is denoted by $\tilde{x}(\tilde{t})$. Note that an arbitrary position on the table can be chosen as the origin of $\tilde{x}(\tilde{t})$. Between the table and the mass $\tilde{m}$, a Coulomb friction force $\tilde{F}_{f}$ with friction coefficient $\mu$ exists, which mathematically can be described as follows (see e.g. [11]):

$$
\tilde{F}_{f} \in \mu \tilde{m} \tilde{g} \operatorname{Sign}(\dot{\tilde{x}})
$$

where $\tilde{g}$ is the acceleration due to gravity, and $\operatorname{Sign}(\dot{\tilde{x}})$ is the set-valued sign function, defined by:

$\operatorname{Sign}(\dot{\tilde{x}})= \begin{cases}\{1\} & \text { if } \dot{\tilde{x}}>0 \\ {[-1,1]} & \text { if } \dot{\tilde{x}}=0 \\ \{-1\} & \text { if } \dot{\tilde{x}}<0\end{cases}$

Note that in case the mass sticks to the table (so $\dot{\tilde{x}}=0$ ), the friction force can take values in the range $[-\mu \tilde{m} \tilde{g}, \mu \tilde{m} \tilde{g}]$. This allows the mass to stick to the table as long as the external force acting on the mass is small enough. Using Newton's second law it is straightforward to derive the equation of motion for the mass:

$-\tilde{F}_{f}=\tilde{m}(\ddot{\tilde{x}}+\ddot{\tilde{y}})$

If the relative velocity of the mass is zero $(\dot{\tilde{x}}=0)$ and the external force acting on the mass is small enough (i.e. $|\tilde{m} \ddot{\tilde{y}}|<\mu \tilde{m} \tilde{g}$ ), the friction force will balance this external force $\left(-\tilde{F}_{f}=\tilde{m} \ddot{\tilde{y}}\right)$ resulting in $\ddot{\tilde{x}}=0$, i.e. in sticking of the mass. If the external force is larger than the maximum friction force (i.e. $|\tilde{m} \ddot{\tilde{y}}|>\mu \tilde{m} \tilde{g})$, the mass will start to slip, resulting in $\dot{\tilde{x}} \neq 0$ and $\tilde{F}_{f}=\mu \tilde{m} \tilde{g} \operatorname{Sign}(\dot{\tilde{x}})$.

\subsection{Dimensionless dynamical model}

The equation of motion derived in the previous section will be made dimensionless to obtain a model which incorporates a minimum number of parameters.
A characteristic length scale $\tilde{L}$ and a characteristic time scale $\tilde{T}$ need to be introduced. The dimensionless length scales $x$ and $y$ and dimensionless time scale $t$ are defined in the following way:

$x=\tilde{x} / \tilde{L}, \quad y=\tilde{y} / \tilde{L}, \quad t=\tilde{t} / \tilde{T}$

Also, a dimensionless differential operator is defined as ' $=d / d t$. If we choose $\tilde{T}=\sqrt{\tilde{L} / \mu \tilde{g}}$, the equation of motion (3) can be written in dimensionless form as:

$-F_{f}=x^{\prime \prime}+y^{\prime \prime}$

where $F_{f}=\tilde{F}_{f} / \mu \tilde{m} \tilde{g} \in \operatorname{Sign}\left(x^{\prime}\right)$, using (1). Note that (5) can now be written as:

$-\operatorname{Sign}\left(x^{\prime}\right) \ni x^{\prime \prime}+y^{\prime \prime}$

The dimensionless condition for sticking of the mass $\left(x^{\prime}=0\right)$ now reduces to $\left|y^{\prime \prime}\right|<1$.

\subsection{Stopping region}

One of the objectives is to accurately stop the mass at a desired end location. To achieve this, an increase in friction coefficient is used near the desired end location $x=x_{d}$ of the mass. In essence, the table will consist of two regions, a low friction region and a high friction region. The high friction region will start at position $x=x_{\mu}$, see Fig. 2. The low friction region has a friction coefficient $\mu_{1}=\mu$ and the high friction region has a friction coefficient $\mu_{2}=c_{\mu} \mu$, with $c_{\mu}>1$.

Because the mass has a finite dimensionless width $w=\tilde{w} / \tilde{L}(\tilde{w}$ is the width in SI units), it will not experience a discontinuous step in the friction coefficient, when it enters the high friction region. More realistically, in the transition region, the mass will experience an effective friction coefficient $\bar{\mu}$, depending on 
Fig. 2 Schematic of the table with two regions, one region with low friction coefficient $\mu_{1}$ and another region with high friction coefficient $\mu_{2}$

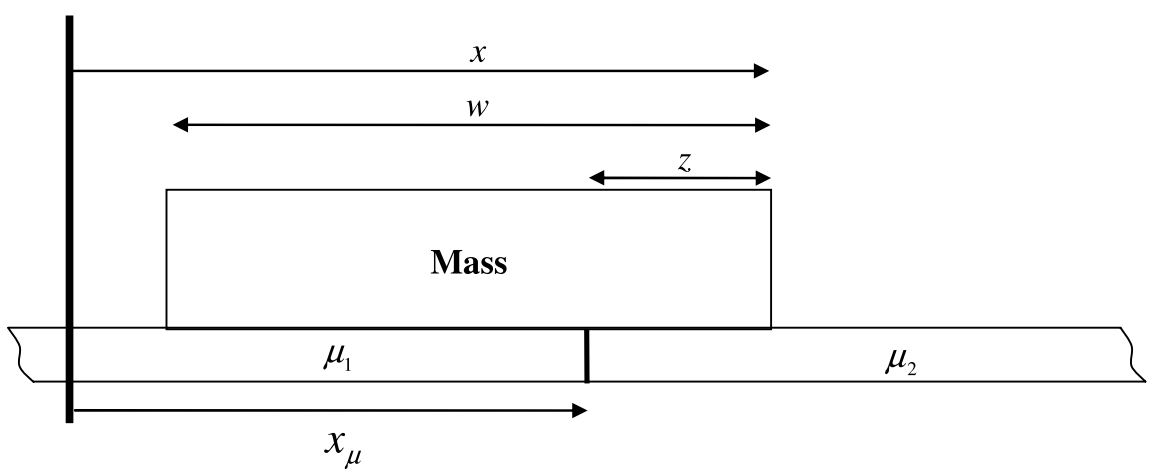

the distribution of the weight of the mass over the low friction region and the high friction region:

$\bar{\mu}(z)=\frac{w-z}{w} \mu_{1}+\frac{z}{w} \mu_{2}=\frac{w+\left(c_{\mu}-1\right) z}{w} \mu$

where $z$ is the portion of the mass on the high friction region, see Fig. 2. Note that $z$ is bounded by $0 \leq z \leq w$. This constraint can conveniently be formulated using a min-max formulation:

$z=\min \left(\max \left(x-x_{\mu}, 0\right), w\right)$

The friction coefficient $\bar{\mu}$ that the mass experiences, thus depends on the position $x$ of the mass. Note that in the transition region, using (7) and (8), the friction coefficient can be written as follows:

$$
\begin{gathered}
\bar{\mu}(x)=\mu\left(1+\frac{c_{\mu}-1}{w}\left(x-x_{\mu}\right)\right) \\
\text { if } x_{\mu} \leq x \leq x_{\mu}+w
\end{gathered}
$$

In all simulation results presented throughout this paper, it is assumed that the mass does not tip over, i.e. it is assumed that the mass will remain in full contact with the table, so that the equation of motion (6) remains valid. In general, this will be valid for an object the height of which is relatively small with respect to its width $w$.

\section{Table trajectory design}

The design of the table motion profile will be discussed in this section. First, in Sect. 4.1, a simulation method called the time-stepping method will be discussed. In Sect. 4.2, an example of a stick-slip motion of the mass, calculated using the time-stepping method, will be shown. An objective function will be introduced in Sect. 4.3, which is used in Sect. 4.4 to design a suitable table excitation signal.

\subsection{Simulation method}

The system under study exhibits dry friction, which makes it a nonlinear system with unilateral constraints [12]. There are different methods that can be used to simulate these kind of systems. Time-stepping is an efficient method for numerically solving the equations describing the dynamics of systems with unilateral constraints. A thorough mathematical description of time-stepping method can be found in [11] and [22]. In contrast to, for example, event-driven techniques, in time-stepping it is not necessary to detect every event (e.g. a stick-slip transition). The solution is computed using fixed time-steps forward in time. The time-stepping method of Moreau [14] is used as the integration routine in this paper. A fixedpoint iteration is carried out to solve for the unknown velocity in the system at every time-step. This velocity is used to estimate the position at the end of the time-step.

\subsection{Example of stick-slip motion}

An example of a simulated response using timestepping is shown in Fig. 3. Note that the timestepping routine does not explicitly solve for the acceleration $x^{\prime \prime}(t)$ of the mass. A periodic excitation signal consisting of a sequence of second order polynomials was initially used for the table displacement signal $y(t)$. Intuitively, the table should move slowly in the desired direction dragging the mass along, and fast in the opposite direction, resulting in slipping of 
Fig. 3 Simulation of the system using the time-stepping approach ( $x$ : object, $y:$ table)
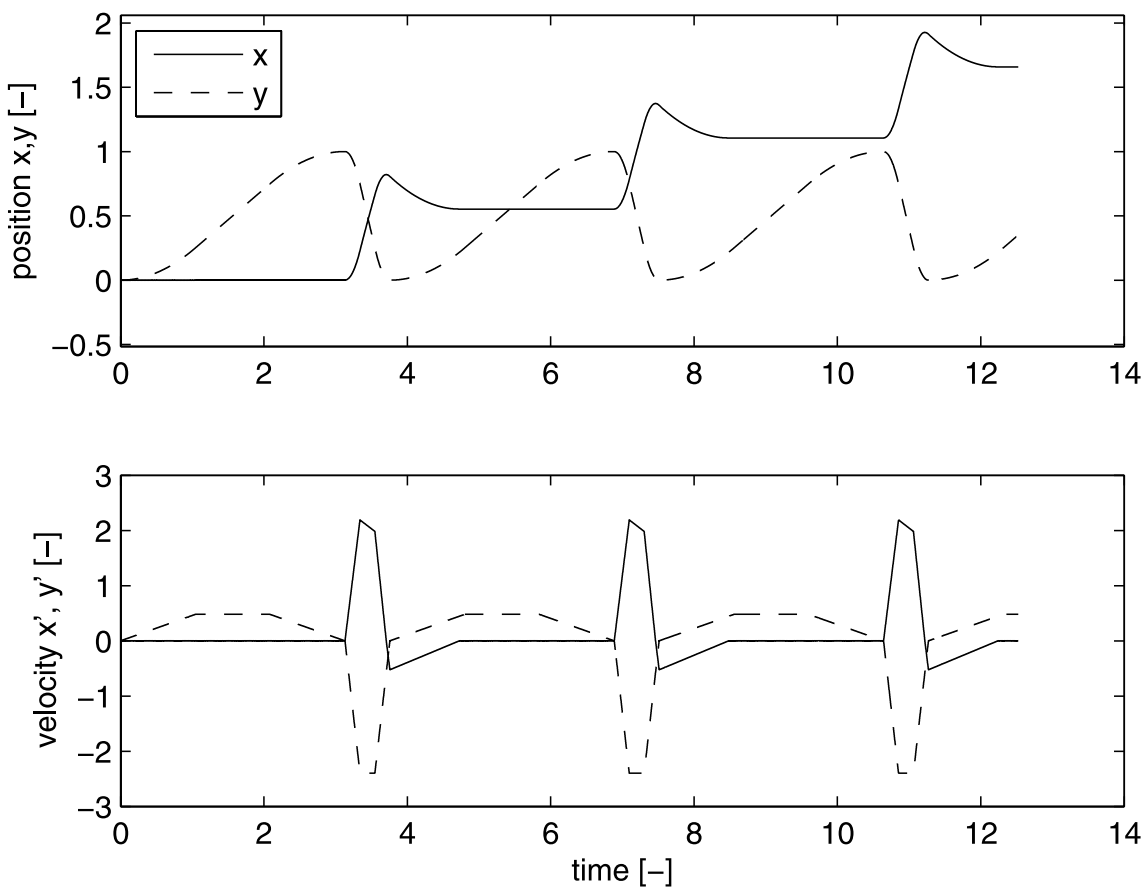

the mass. Figure 3 shows that the principle of moving a mass in a desired direction using stick-slip vibrations works. However, the shape of the prescribed table displacement signal $y(t)$ may be far from optimal. Therefore, the focus will be on the design and optimization of the actuation signal based on an objective function, which will be defined in Sect. 4.3.

\subsection{Objective function}

In order to study the effectiveness of a certain prescribed periodic table trajectory, a sensible choice has to be made for an objective function. In conformity with the first objective from Sect. 2, the mass should move in one direction as fast as possible, meeting certain actuator constraints related to stroke and excitation frequency. An appropriate quantity to use in an objective function will therefore be the average velocity $\bar{v}$ of the mass in steady state (in the $\mu_{1}$ region). Consequently, transient behavior is not considered. This average velocity $\bar{v}$ can be written as:

$\bar{v}=\lim _{t \rightarrow \infty} \frac{x(t+\Delta t)-x(t)}{\Delta t}$

where $\Delta t$ is the period time of the periodic relative velocity signal of the mass. Obviously, in the case of pure stick, which is undesirable in the motion phase, $\bar{v}$ will be zero. The following objective function $f$ is introduced:

$f=\frac{1}{c \bar{v}^{2}}$

where the positive constant $c=10,000$ is used for normalizing the objective function values throughout this paper. Obviously, this constant in principle has no influence on the optimization process itself. Minimizing $f$ is equivalent to maximizing the average steadystate velocity $\bar{v}$ and to minimizing the process time $T_{p}=\tilde{T}_{p} / \tilde{T}$ (neglecting transients effects).

\subsection{Design of the table motion profile}

From (6) it is clear that the trajectory of the table directly influences the dynamics of the mass through $y^{\prime \prime}(t)$. Because the excitation of the table will be periodic, the stroke of the table $\Delta y=\max (y)-\min (y)$ and the excitation frequency $1 / \Delta t$ will be two important parameters that influence the dynamics. In the following, the influence of these two parameters, $\Delta y$ and $\Delta t$, is studied and the design of the shape of the trajectory profile of the table (which completely specifies $\left.y^{\prime \prime}(t)\right)$ is examined. 
Fig. 4 Influence of excitation frequency, objective function value $f$ vs. $\Delta t$

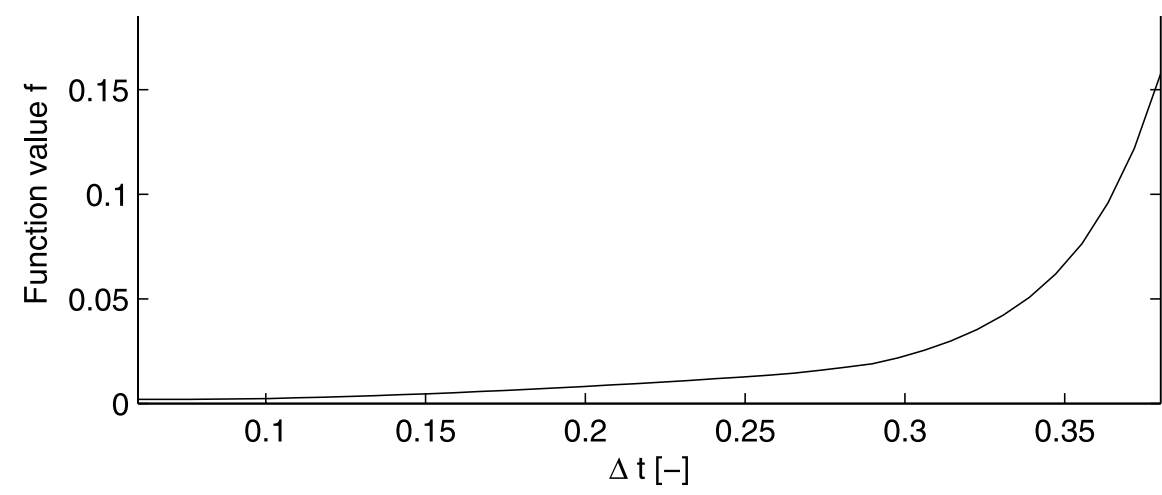

Fig. 5 Influence of stroke, objective function value $f$ vs. $\Delta y$

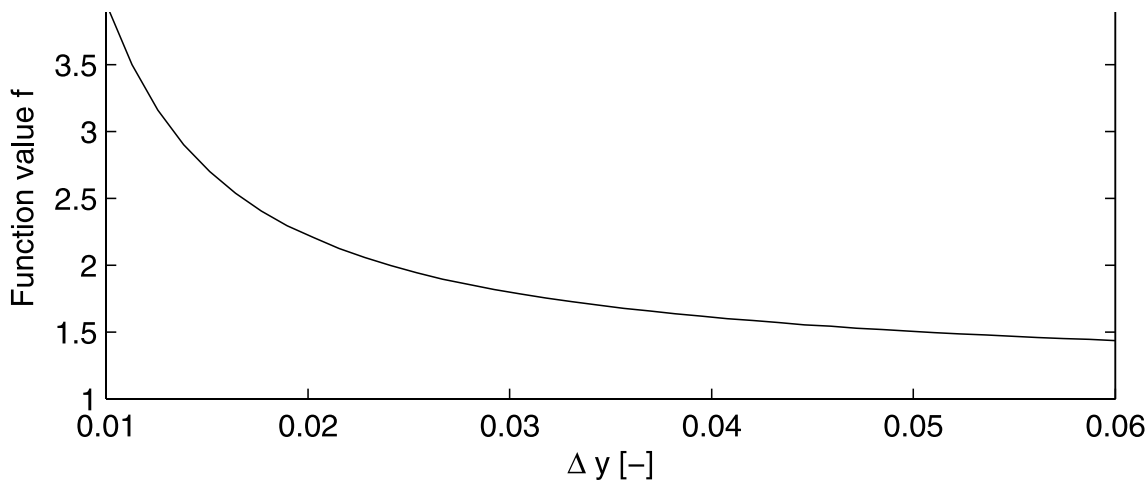

\subsubsection{Preliminary study of influence of excitation frequency and stroke}

The excitation frequency $1 / \Delta t$ and stroke $\Delta y$ both have a strong influence on the average steady-state velocity of the mass. Here, again, a sequence of second order polynomials similar to $y(t)$ in Fig. 3 is used for the table motion profile along with the time-stepping simulation routine. In Figs. 4 and 5 the objective function value $f$ defined by (11) is plotted as a function of $\Delta t$ (for $\Delta y=0.02$ ) and $\Delta y$ (for $\Delta t=0.05$ ), respectively. It is clear that for this type of table trajectories and the values given above, a large excitation frequency and a large stroke are both beneficial in obtaining a large average steady-state velocity $\bar{v}$ of the mass. Of course, suitable values for $\Delta t$ and $\Delta y$ will depend on the application in mind and on actuator constraints.

\subsubsection{Preliminary analysis of a Fourier-based motion profile}

Once the values of excitation frequency and stroke are fixed, there is still freedom in designing the precise shape of the table trajectory. The shape of a table motion profile parameterized using a Fourier series of twelfth order has been optimized by minimizing the objective function value $f$, see (11), using constrained gradient based optimization of the Fourier coefficients. The converged result is shown in Fig. 6. The following settings have been applied: $\Delta t=0.072, \Delta y=0.021$, $y_{\max }^{\prime}=3.2$, and $y_{\max }^{\prime \prime}=1275$.

From Fig. 6 it is clear that the optimization procedure converged to a table motion profile, which in approximation has the following nature:

- a time interval with zero acceleration and constant nonzero velocity;

- a time interval with constant negative acceleration values;

- a time interval with constant positive acceleration values.

It seems that the largest part of the time interval with approximately zero-acceleration in approximation results in sticking of the mass to the table. This effect is clearly visible in Fig. 6: in steady state, the mass (almost) does not slide back, i.e. it does not move in the undesired direction. These observations are the 
Fig. 6 Converged optimization of the Fourier signal using constrained gradient based optimization, $f=56$
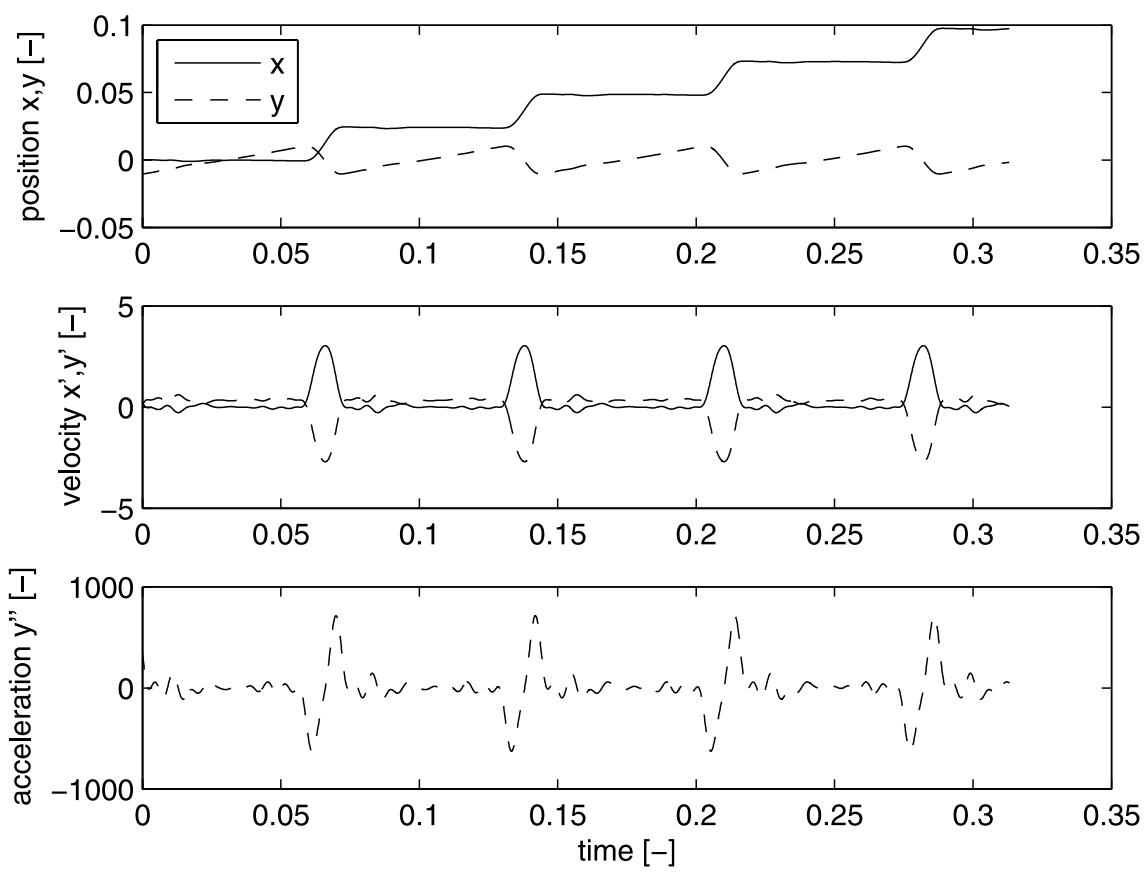

motivation for the design of a table trajectory generator incorporating these three distinct time intervals with constant acceleration values. This will be discussed in detail in the next subsection.

\subsubsection{Prescribed table motion with three constant acceleration time intervals}

The prescribed table motion with three constant acceleration time intervals will consist of: a time interval with zero acceleration, a time interval with constant negative acceleration and a time interval with constant positive acceleration. This is, in approximation, the signal which was obtained after optimization in the previous subsection. As stated before, for practical reasons, the table should return to the same position after each excitation period. As indicated in Fig. 7, the following 9 parameters determine the shape of the table trajectory: $y_{1}^{\prime \prime}, y_{2}^{\prime \prime}, y_{3}^{\prime \prime}, t_{0}, t_{1}, t_{2}, t_{3}, y_{\text {ini }}$, and $y_{\text {ini }}^{\prime}$. There are some constraints to the table motion profile that need to be satisfied:

1. Minimal table displacement should be 0 (this is a choice rather than a constraint);

2. $t_{0}=0$ (this is also a choice rather than a constraint);

3. Fix the period time $\Delta t$;

4. Fix the stroke $\Delta y$;
5. The start position should be equal to the end position (periodicity requirement, in the second subplot of Fig. 7 the shaded area below the zero axis should be equal to the shaded area above the zero axis);

6. The start velocity should be equal to the end velocity (periodicity requirement, in the third subplot of Fig. 7 the shaded area below the zero axis should be equal to the shaded area above the zero axis);

7. $y_{1}^{\prime \prime}=0$.

Thus, two parameters can be chosen freely, for example $y_{2}^{\prime \prime}$ and $y_{3}^{\prime \prime}$. Subsequently, the trajectory generator needs to determine five unknowns: the times $t_{1}, t_{2}$, $t_{3}$, the initial position $y_{\text {ini }}$, and the initial velocity $y_{\text {ini }}^{\prime}$. The mathematical description of the constraints and the trajectory generator algorithm can be found in Appendix. In general, parameter values will be chosen such that certain phenomena can be clearly observed and explained.

\section{Analysis of steady-state response}

The analytic steady-state solutions of the mass and the classification of these solutions, using the prescribed table motion with three acceleration parts, will be addressed in Sect. 5.1. The results of some parameter studies will be discussed in Sect. 5.2. 


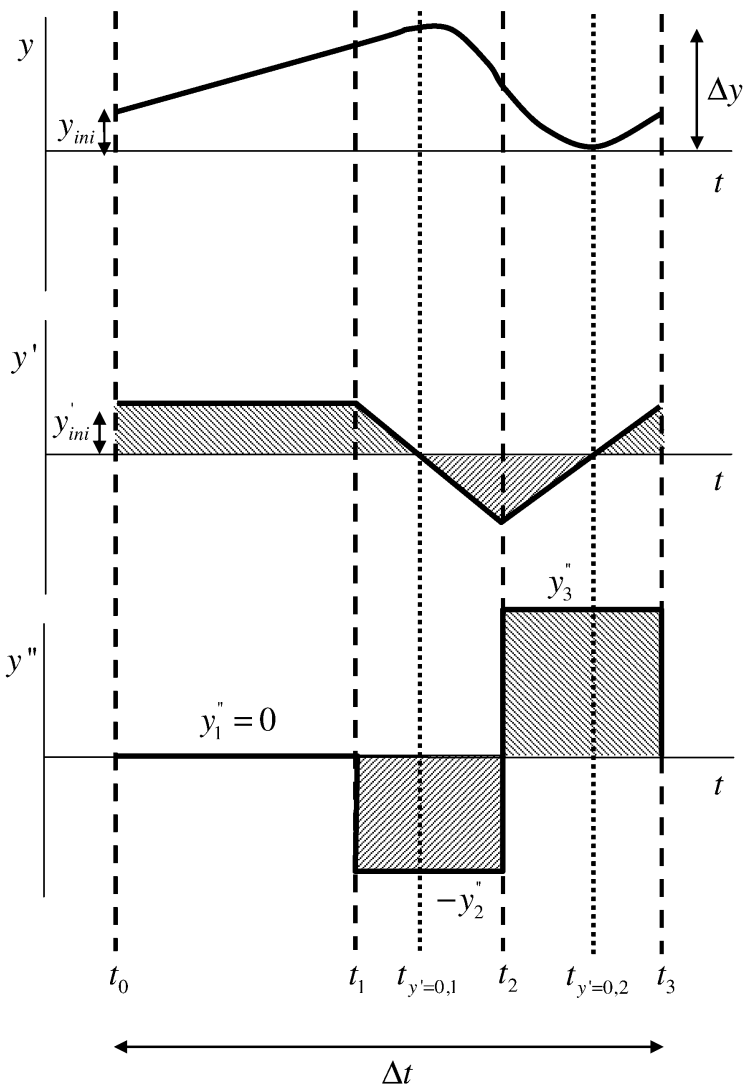

Fig. 7 Sketch of the table motion profile, which consists of three time intervals with constant acceleration

\subsection{Analytic solutions and classification}

Given a prescribed motion profile of the table obtained in Sect. 4.4.3, the equation of motion (6) can be solved analytically by splitting the solution into different time intervals and combining them in one general solution by requiring continuity of $x(t)$. This allows for a classification of different types of mass motions. The analytic solution will be discussed in detail here for one type of motion only.

For the case considered here, $y_{2}^{\prime \prime}>1$ and $y_{3}^{\prime \prime}<1$, which means that the mass cannot stick in $\left[t_{1}, t_{2}\right]$ but can stick in $\left[t_{2}, t_{3}\right]$. Note that the accelerations are defined in such a way that $y_{2}^{\prime \prime}>0$ and $y_{3}^{\prime \prime}>0$, see Fig. 7 . Every time the table acceleration values change (at times $\left.t_{1}, t_{2}, t_{3}\right)$ and when the relative velocity $x^{\prime}$ becomes zero, a discontinuity occurs in the mass acceleration $x^{\prime \prime}$, such that the solution needs to be determined for all these time spans separately. Starting with $x_{0}^{\prime}=x_{0 s}^{\prime}=0$ will yield a steady-state trajectory imme- diately, as will be shown. In the remainder of this paper, if the initial conditions are chosen such that these yield a steady-state trajectory from the start (corresponding to the correct phase of the table motion), this will be denoted by the subscript ' $s$.' First, consider the time span $\left[t_{0}, t_{1}\right]$. The table acceleration is zero in this time span, so the mass will stick to the table. The relative velocity and acceleration of the mass will thus be zero. Assuming that the initial position of the mass is also zero, gives for $t_{0} \leq t \leq t_{1}$ :

$$
\begin{aligned}
& x^{\prime \prime}(t)=0, \\
& x^{\prime}(t)=0, \\
& x(t)=0
\end{aligned}
$$

In the second time interval $\left[t_{1}, t_{2}\right]$, the mass will start to slip because $y_{2}^{\prime \prime}>1$. Note that from (6) it follows that the acceleration of the mass becomes $x^{\prime \prime}=$ $-1+y_{2}^{\prime \prime}$, so for $t_{1} \leq t \leq t_{2}$ :

$$
\begin{aligned}
& x^{\prime \prime}(t)=-1+y_{2}^{\prime \prime}, \\
& x^{\prime}(t)=\left(-1+y_{2}^{\prime \prime}\right)\left(t-t_{1}\right), \\
& x(t)=\left(-1+y_{2}^{\prime \prime}\right)\left(t-t_{1}\right)^{2} / 2
\end{aligned}
$$

At time instance $t_{2}$, the acceleration changes from $-y_{2}^{\prime \prime}$ to $y_{3}^{\prime \prime}$. Therefore, the acceleration of the mass changes to $x^{\prime \prime}=-1-y_{3}^{\prime \prime}$. For $t_{2} \leq t \leq t_{x^{\prime}=0}$ :

$$
\begin{aligned}
x^{\prime \prime}(t)= & -1-y_{3}^{\prime \prime}, \\
x^{\prime}(t)= & x^{\prime}\left(t_{2}\right)+\left(-1-y_{3}^{\prime \prime}\right)\left(t-t_{2}\right), \\
x(t)= & x\left(t_{2}\right)+x^{\prime}\left(t_{2}\right)\left(t-t_{2}\right) \\
& \quad+\left(-1-y_{3}^{\prime \prime}\right)\left(t-t_{2}\right)^{2} / 2
\end{aligned}
$$

where $t_{x^{\prime}=0}$ is the time at which the relative velocity of the mass $x^{\prime}$ becomes zero because $y_{3}^{\prime \prime}<1$. This time can be calculated analytically:

$t_{x^{\prime}=0}=t_{2}+\frac{-x^{\prime}\left(t_{2}\right)}{-1-y_{3}^{\prime \prime}}$

The mass will stick until $t=t_{3}$. Indeed, the initial condition $x_{0 s}^{\prime}=0$ leads to a steady-state trajectory. Note that in principle only the velocity and acceleration of the mass are in steady state, in contrast to the position, because the mass has a net nonzero displacement every period time $\Delta t=t_{3}-t_{0}$. 
For $t_{x^{\prime}=0} \leq t \leq t_{3}$ :

$x^{\prime \prime}(t)=0$,

$x^{\prime}(t)=0$,

$x(t)=x\left(t_{x^{\prime}=0}\right)$

Simulations and solving different cases analytically point out that in the $\mu_{1}$ region 5 different types of steady-state responses can occur, depending on the magnitudes of $y_{2}^{\prime \prime}$ and $y_{3}^{\prime \prime}$, see Table 1 .

Note that the analytic solution for case 1 has been derived above. For cases $1-4$, the period of the relative velocity response is equal to the excitation period $\Delta t$.

The analytic solutions of cases $2-5$ can be derived in an equivalent manner as was done above for the case 1 type of motion. Determination of the resulting case can be done a priori by following the flow

Table 1 Table showing possible types of steady-state responses

\begin{tabular}{lll}
\hline Case & Description & (Un)desirable? \\
\hline 1 & Stick-slip with & Desirable \\
& $x^{\prime}(t) \geq 0, \forall t$ & \\
2 & Stick-slip with & Undesirable \\
& $x^{\prime}(t) \leq 0, \forall t$ & \\
3 & General stick-slip & Acceptable if $\bar{v}>0$ \\
4 & Slip-slip & Acceptable if $\bar{v}>0$ \\
5 & Pure stick & Only desirable \\
& (no relative motion) & in stopping region \\
\hline
\end{tabular}

chart displayed in Fig. 8. Figure 9 gives an illustrative graphical overview of the 5 cases that can occur when the table motion with three constant acceleration time intervals is used. In addition, a non-physical, complex table trajectory may be obtained if, for example, a very large stroke $\Delta y$ is requested in a very short time span $\Delta t$ using very small acceleration values $y_{2}^{\prime \prime}$ and $y_{3}^{\prime \prime}$. This non-physical table trajectory will be denoted by case 0 .

\subsection{Parameter studies and objective function evaluation}

The analytic solutions of the mass motion resulting from the prescribed periodic table motion, can be used for very fast and exact parameter studies. In this way, it is possible to make surface plots of the objective function value $f$, see (11), as a function of $y_{2}^{\prime \prime}$ and $y_{3}^{\prime \prime}$. There is a strong analogy between the case of motion that is occurring and the objective function value. In Fig. 10, the average steady-state velocity $\bar{v}$ of the mass drops dramatically ( $f$ dramatically increases), if the type of motion changes from a general stickslip motion to a slip-slip type of motion. As a design rule, this would mean that slip-slip trajectories should be avoided. Objective function values are limited to $f=1.6 \times 10^{-3}$ for clarity. Figure 11 considers a range of acceleration values that are much smaller, such that stick can occur in time intervals of nonzero acceleration (case 1 or case 2 solutions). Also, there
Fig. 8 Flow chart to calculate a priori which case will occur

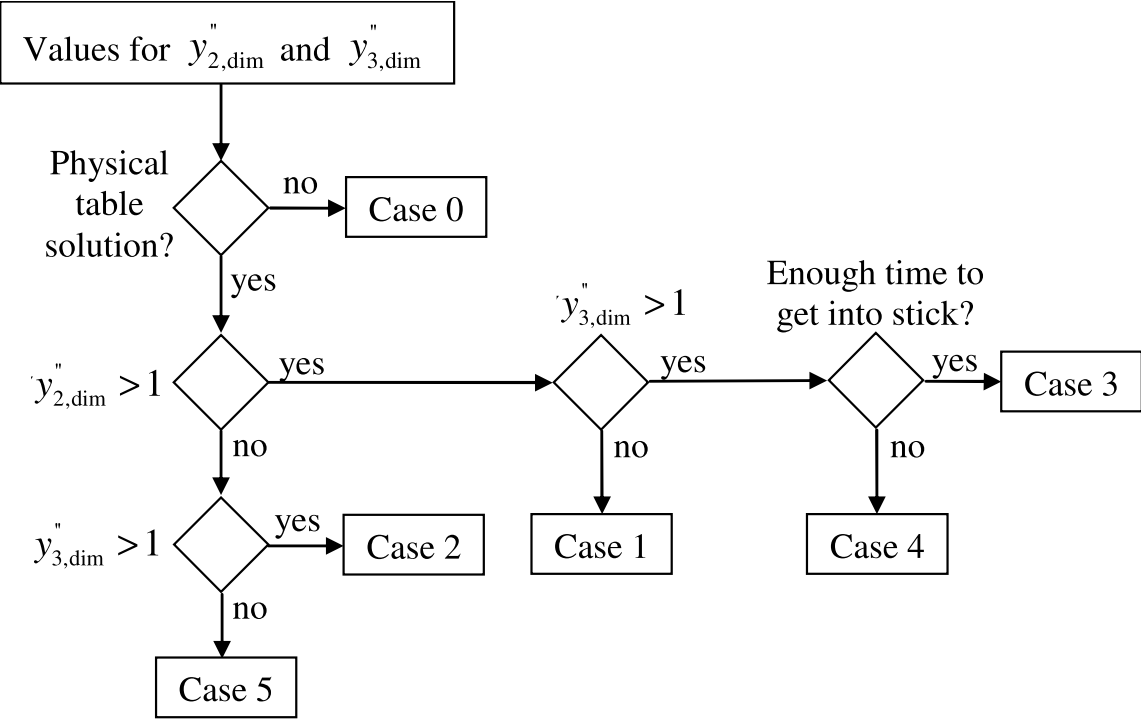


Fig. 9 Examples of time histories of the 5 different cases that can occur
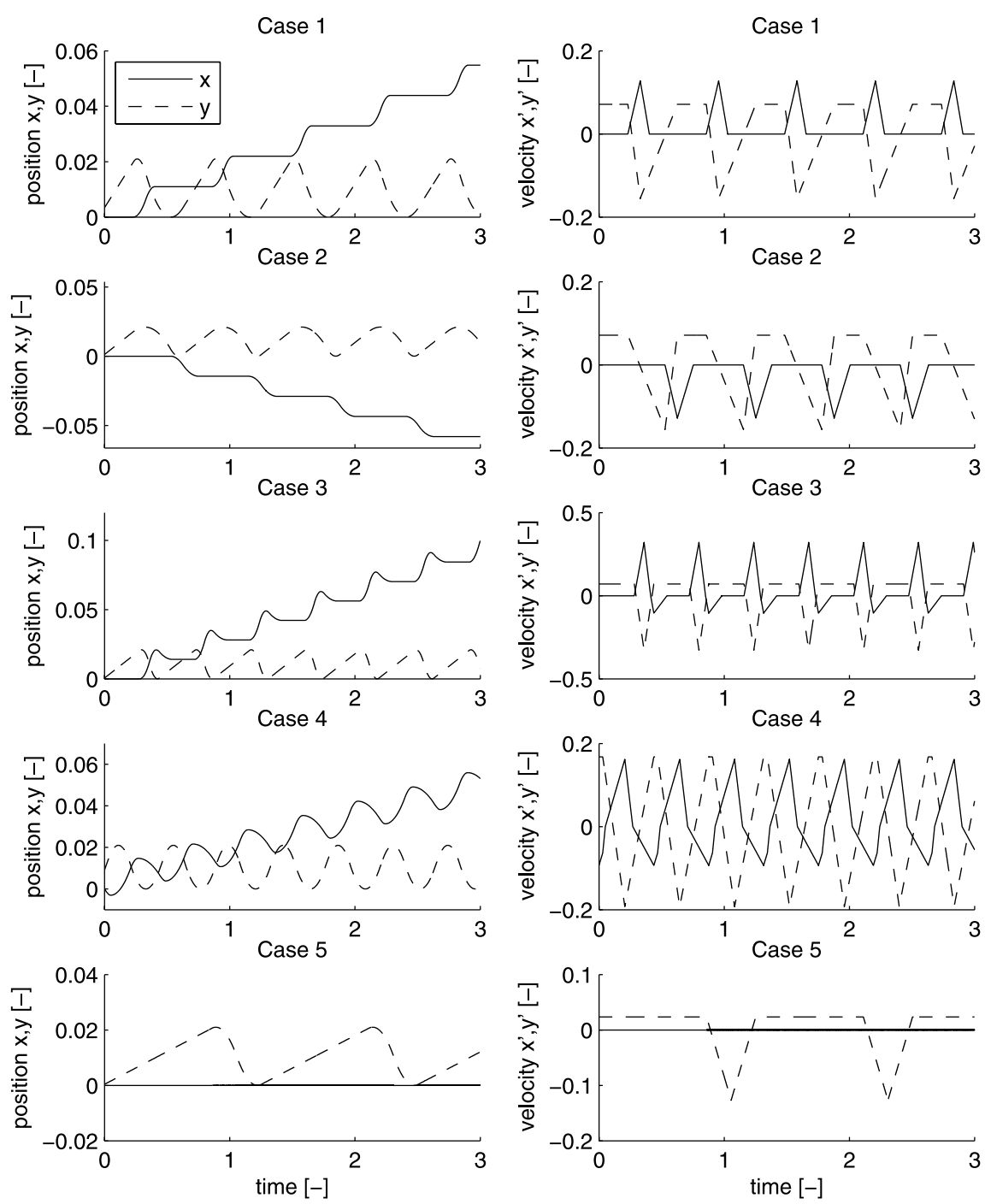

is a clear relation between the type of motion and the average steady-state velocity. Case 1 solutions seem preferable. Objective function values $f \geq 0.8$ are set to $f=0.8$ for clarity.

The design rules for making a trajectory, for the current system and studied parameter ranges, can be summarized as follows:

- Increase the frequency of excitation (subject to practical constraints);

- Increase the stroke (subject to practical constraints);

- Use surface plots to make a sensible choice for the acceleration values $y_{2}^{\prime \prime}$ and $y_{3}^{\prime \prime}$ such that the chosen settings are not too sensitive. The response should be a case 1 (stick-slip, $x^{\prime} \geq 0$ ), a case 3 (general stick-slip), or a case 4 (slip-slip) response; the latter two cases are only acceptable if $\bar{v}>0$. Case 2 (stick-slip, $x^{\prime} \leq 0$ ) and case 5 (just stick) responses are prohibited.

\section{Local stability and convergence}

\subsection{Local stability analysis}

Using Floquet theory, it can be proven that all steadystate solutions from Sect. 5.1 are locally stable. Three different approaches have been used to calculate the Floquet multipliers: (1) an analytical method based 
Fig. 10 Surface plot and case plot for the settings $\Delta y=0.021, \Delta t=0.072$

Fig. 11 Surface plot and case plot for the settings $\Delta y=0.064, \Delta t=1.38$
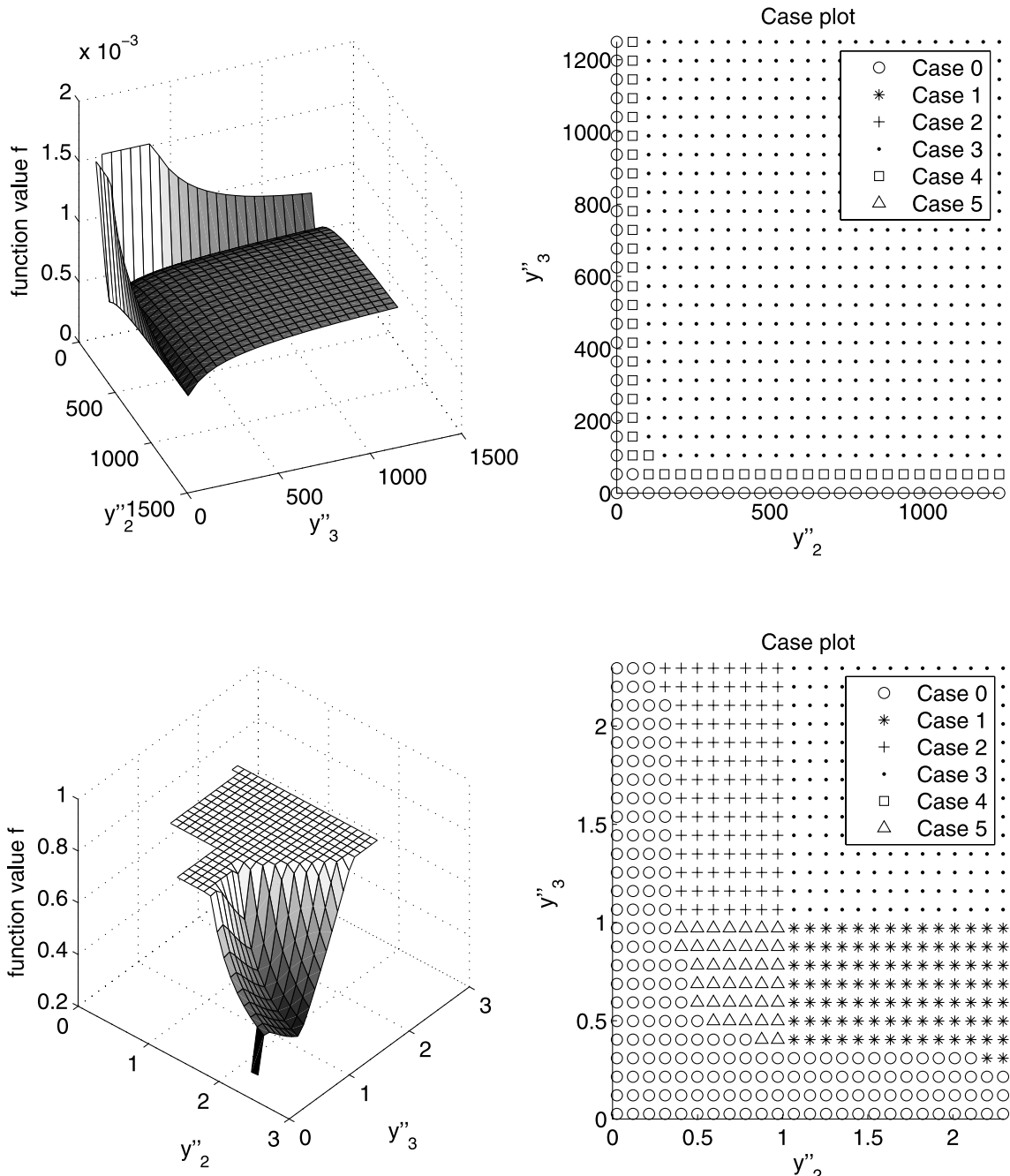

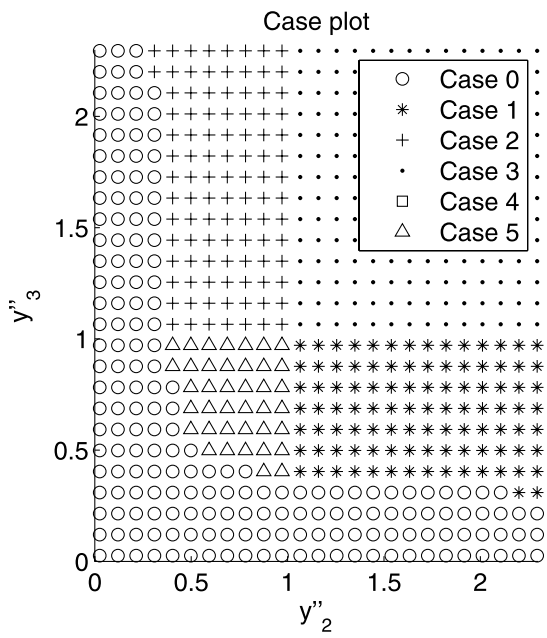

on linearization of the effects of the perturbations of the analytic steady-state solutions over a complete period, (2) a second analytical method, in which the Monodromy matrix is calculated by coupling smooth parts of the Fundamental solution matrix using Saltation matrices [11], and (3) a numerical method, which estimates the Monodromy matrix using a sensitivity analysis. Here, the first method will be treated. Local stability is studied using the analytic expressions from Sect. 5.1, and following the same approach as in [15]. Infinitesimally small perturbations $\Delta x_{0}$ and $\Delta x_{0}^{\prime}$ on the initial position (respectively, the initial velocity) are considered. After linearization, perturbations in the position and velocity of the mass at the end of one period, i.e. at $t=t_{3}, \Delta x_{3}$ and $\Delta x_{3}^{\prime}$, are linearly related to the perturbations in initial position $\Delta x_{0}$ and velocity $\Delta x_{0}^{\prime}$ as follows:

$\left[\begin{array}{l}\Delta x_{3} \\ \Delta x_{3}^{\prime}\end{array}\right]=\Phi\left(t_{3}, t_{0}\right)\left[\begin{array}{l}\Delta x_{0} \\ \Delta x_{0}^{\prime}\end{array}\right]$

where $\Phi\left(t_{3}, t_{0}\right)$ is the so-called Monodromy matrix. Note that the times $t_{i}$ correspond to the notation of Sect. 4.4.3; see also Fig. 7. For a case 1 response (stick-slip with $\left.x^{\prime}(t) \geq 0, \forall t\right)$ the analytic local stability analysis will be carried out here. An illustrative case 1 response is shown in Fig. 12. The mass will be in steady state at $t=t_{0}$ if the initial velocity is chosen as $x_{0 s}^{\prime}=0$.

Using the analytic expressions derived in Sect. 5.1 and applying the initial conditions $x\left(t_{0}\right)=x_{0 s}$ and 
Fig. 12 Example of an illustrative case 1 trajectory
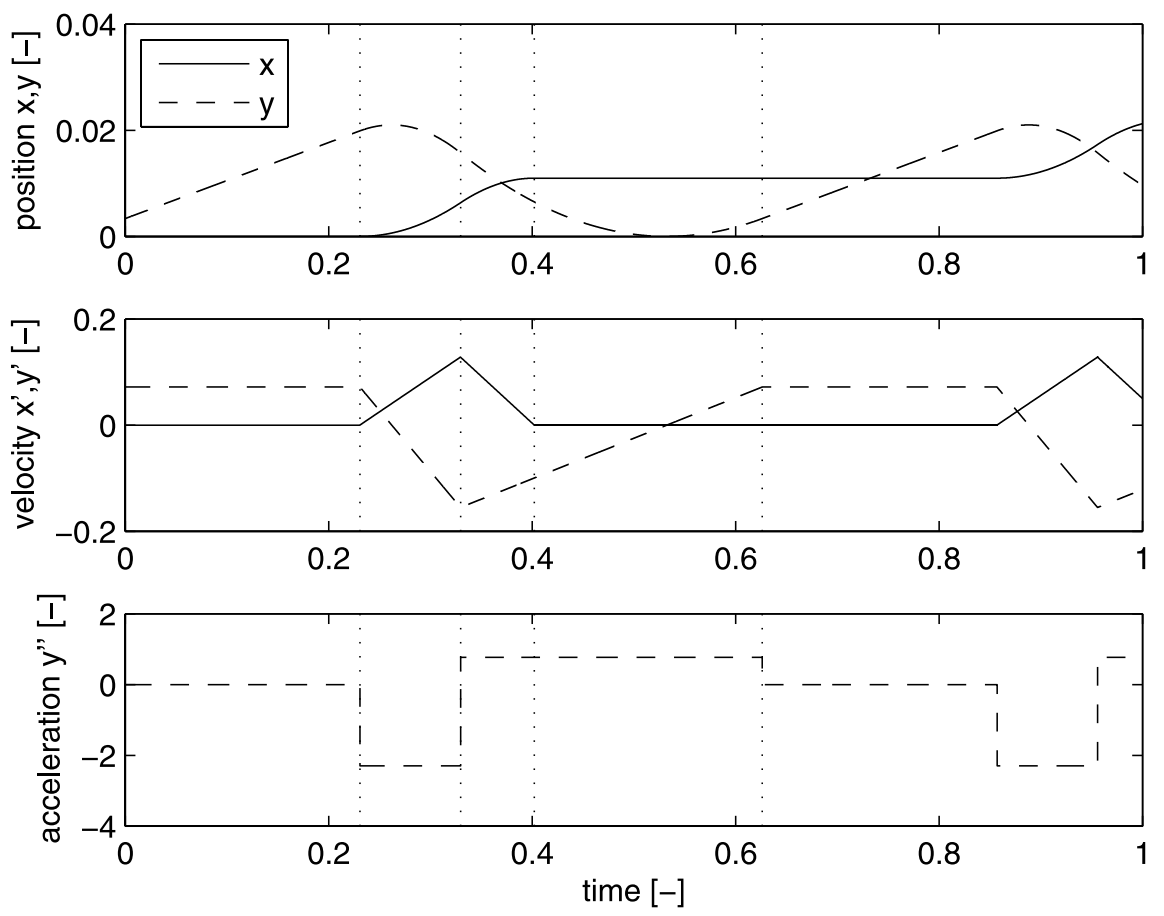

$x^{\prime}\left(t_{0}\right)=x_{0 s}^{\prime}$, it can be shown that the position and velocity at $t=t_{3}$ can be written as:

$x\left(t_{3}\right)=x_{0 s}+x_{0 s}^{\prime 2} / 2+c_{1}$,

$x^{\prime}\left(t_{3}\right)=0$

where $c_{1}$ is a constant. Using $x_{0 s}+\Delta x_{0}$ and $x_{0 s}^{\prime}+\Delta x_{0}^{\prime}$ as initial conditions at $t=t_{0}$ will result in position $x\left(t_{3}\right)+\Delta x_{3}$ and velocity $x^{\prime}\left(t_{3}\right)+\Delta x_{3}^{\prime}$ at $t=t_{3}$. Substitution of these quantities in (26) and (27) gives:

$x\left(t_{3}\right)+\Delta x_{3}=x_{0 s}+\Delta x_{0}+\left(x_{0 s}^{\prime}+\Delta x_{0}^{\prime}\right)^{2} / 2+c_{1}$,

$x^{\prime}\left(t_{3}\right)+\Delta x_{3}^{\prime}=0$

By substituting (26)-(27) in (28)-(29), keeping only the linear terms in $\Delta x_{0}$ and $\Delta x_{0}^{\prime}$, and applying the initial condition $x_{0 s}^{\prime}=0$ (to assess the local stability of the steady-state solution), the following relation between perturbations at $t=t_{0}$ and $t=t_{3}$ can be derived:

$\left[\begin{array}{l}\Delta x_{3} \\ \Delta x_{3}^{\prime}\end{array}\right]=\left[\begin{array}{ll}1 & 0 \\ 0 & 0\end{array}\right]\left[\begin{array}{l}\Delta x_{0} \\ \Delta x_{0}^{\prime}\end{array}\right]$

The Floquet multipliers, i.e. the eigenvalues of the Monodromy matrix, are $\lambda_{1}=1$ and $\lambda_{2}=0$. The eigen- value equal to 1 is expected due to the fact that a perturbation in the initial position only, will only shift the end position by the same amount. The corresponding eigenvector of $\lambda_{1}=1$ is $\mathbf{v}_{1}=[1,0]^{T}$, which confirms that this eigenvalue is purely related to the freedom in position. The second eigenvalue $\lambda_{2}=0$ and the corresponding eigenvector $\mathbf{v}_{2}=[0,1]^{T}$, which illustrates the fact that a small change in initial velocity will not change the end velocity at all because the mass will get into stick anyway, despite the small perturbation at $t=t_{0}$.

For the cases 2 and 3 a completely equivalent reasoning can be used. In both cases, this leads to the same eigenvalues as for case 1. Case 4, the slip-slip case, is different, because the mass never sticks. This means that the end velocity after one period can be influenced by the initial velocity. It can be shown that the position and velocity after one period can be written as:

$x\left(t_{3}\right)=x_{0 s}+c_{1} x_{0 s}^{\prime 2}+c_{2} x_{0 s}^{\prime}+c_{3}$,
$x^{\prime}\left(t_{3}\right)=c_{4} x_{0 s}^{\prime}+c_{5}$

where the $c_{i}$ for $i=1,2, \ldots, 5$ are all constants. The following (linearized) relation describes the evolution 
of small perturbations $\Delta x_{0}, \Delta x_{0}^{\prime}$ on the initial conditions:

$$
\left[\begin{array}{l}
\Delta x_{3} \\
\Delta x_{3}^{\prime}
\end{array}\right]=\left[\begin{array}{cc}
1 & 2 c_{1} x_{0 s}^{\prime}+c_{2} \\
0 & c_{4}
\end{array}\right]\left[\begin{array}{c}
\Delta x_{0} \\
\Delta x_{0}^{\prime}
\end{array}\right]
$$

The Floquet multipliers can be shown to be $\lambda_{1}=1$, which again represents the freedom in position, and $\lambda_{2}=c_{4}=\left(\left(y_{2}^{\prime \prime}-1\right)\left(y_{3}^{\prime \prime}-1\right)\right) /\left(\left(y_{2}^{\prime \prime}+1\right)\left(y_{3}^{\prime \prime}+1\right)\right)$. Note that this eigenvalue is always positive and smaller than 1 (so within the unit circle) because $y_{i}^{\prime \prime}>1$ for $i=2,3$ in the slip-slip case.

The local stability analysis presented in this section has shown that, besides the fact that the response can be shifted in position, the remaining dynamics are all locally asymptotically stable. The analysis using Saltation matrices to compute the Monodromy matrix gives identical results. The method using numerical estimation using a sensitivity analysis gives a good approximation of the exact analytical results.

\subsection{Convergence}

In this subsection, convergence of the relative velocity solutions $x^{\prime}$ will be proven, which also proves that the solution converges to a unique velocity solution in steady state, independent of the initial conditions. The (dimensionless) relative velocity is here denoted by $v=x^{\prime}$. In terms of the relative velocity, the dynamics is described by (see (6)):

$v^{\prime} \in-\operatorname{Sign}(v)-y^{\prime \prime}(t)$

Now consider two trajectories, $v_{1}\left(t, t_{0}, v_{10}\right)$ and $v_{2}\left(t, t_{0}, v_{20}\right)$, with different initial conditions $v_{10}$ and $v_{20}$ at $t=t_{0}$, and consider the following Lyapunov function:

$V\left(v_{1}, v_{2}\right)=\frac{\left(v_{1}-v_{2}\right)^{2}}{2}$

The time derivative of this Lyapunov function along solutions of the system can be written as follows [16]:

$$
\begin{aligned}
V^{\prime}\left(v_{1}, v_{2}\right) & =\left(v_{1}-v_{2}\right)\left(v_{1}^{\prime}-v_{2}^{\prime}\right) \\
& \in-\left(v_{1}-v_{2}\right)\left(\operatorname{Sign}\left(v_{1}\right)-\operatorname{Sign}\left(v_{2}\right)\right) \\
& \leq 0
\end{aligned}
$$

If $v_{1}$ and $v_{2}$ are both positive or both negative, then $V^{\prime}=0$. Therefore, $V^{\prime}$ is only negative semi-definite and convergence is not proven yet. Let $\hat{v}_{1} \in \operatorname{Sign}\left(v_{1}\right)$ and $\hat{v}_{2} \in \operatorname{Sign}\left(v_{2}\right)$, see (2). Now, it can be shown that if $\hat{v}_{1}=\hat{v}_{2}$ and $v_{1}\left(t_{A}\right) \neq v_{2}\left(t_{A}\right)$ at a certain time $t=t_{A}$ (otherwise convergence has already occurred), there will always be a time $t_{B}>t_{A}$, for which $\hat{v}_{1} \neq \hat{v}_{2}$, resulting in further decrease of $V$. Consider the situation in which $\hat{v}_{1}=\hat{v}_{2}$. For both trajectories the acceleration $v_{i}^{\prime}$ will be equal, see (34). Both trajectories will eventually reach zero velocity $v_{i}=0$. To see this, consider (34). The excitation signal $y^{\prime \prime}(t)$ is periodic and over a single period $\Delta t$ it has an average acceleration equal to zero, such that its net effect on the velocity $x^{\prime}$ of the mass over one period time $\Delta t$ is zero. This is shown by integrating (34) over one period time $\Delta t$, for $v \neq 0$ :

$$
\begin{aligned}
v(t+\Delta t)-v(t) & =-\int_{t}^{t+\Delta t}\left(\operatorname{Sign}(v)+y^{\prime \prime}(t)\right) d t \\
& =-\int_{t}^{t+\Delta t} \operatorname{Sign}(v) d t
\end{aligned}
$$

The term $-\operatorname{Sign}(v)$ will force the relative velocity of the mass to $v=0$, which will occur at some point in time. After the trajectory reaches $v=0$, the mass can stick or slip, depending on the value of the acceleration $y^{\prime \prime}$ of the table. At the moment, one of the trajectories reaches $v=0, \hat{v}_{1} \neq \hat{v}_{2}$, and $V^{\prime}<0$, which means that $v_{1}$ and $v_{2}$ will approach each other. This will last until a point in time is reached at which $\hat{v}_{1}=\hat{v}_{2}$ again, and the whole process will repeat itself. Eventually, for cases 1-3 solutions, in finite time a point will be reached at which $v_{1}=v_{2}=0$. From this time onwards, both velocity signals are fully converged, i.e. are equal to each other. In case of a case 4 solution, the mass will never stick, and $v_{1}=v_{2}=0$ will be reached in the limit $t \rightarrow \infty$.

As an example to illustrate this convergence of the velocity solutions, consider Fig. 13. The upper plot of Fig. 13 shows two transients leading to a stick-slip trajectory (case 1). The lower plot of Fig. 13 shows the corresponding Lyapunov function $V$ as a function of time. In Fig. 13, time intervals in which $\hat{v}_{1}=\hat{v}_{2}$ are indicated by ' $=$ '-signs. Time intervals in which $\hat{v}_{1} \neq \hat{v}_{2}$ are indicated by ' $\neq$ '-signs. Clearly, in the time intervals where $\hat{v}_{1} \neq \hat{v}_{2}$, the solutions converge to each other and $V^{\prime}<0$. In the time intervals where $\hat{v}_{1}=\hat{v}_{2}$, the solutions 'run in parallel' and $V^{\prime}=0$. The velocity solutions become identical in the last time interval in Fig. 13. The Lyapunov function $V$ therefore becomes zero. 
Fig. 13 Illustration of convergence of velocity solutions
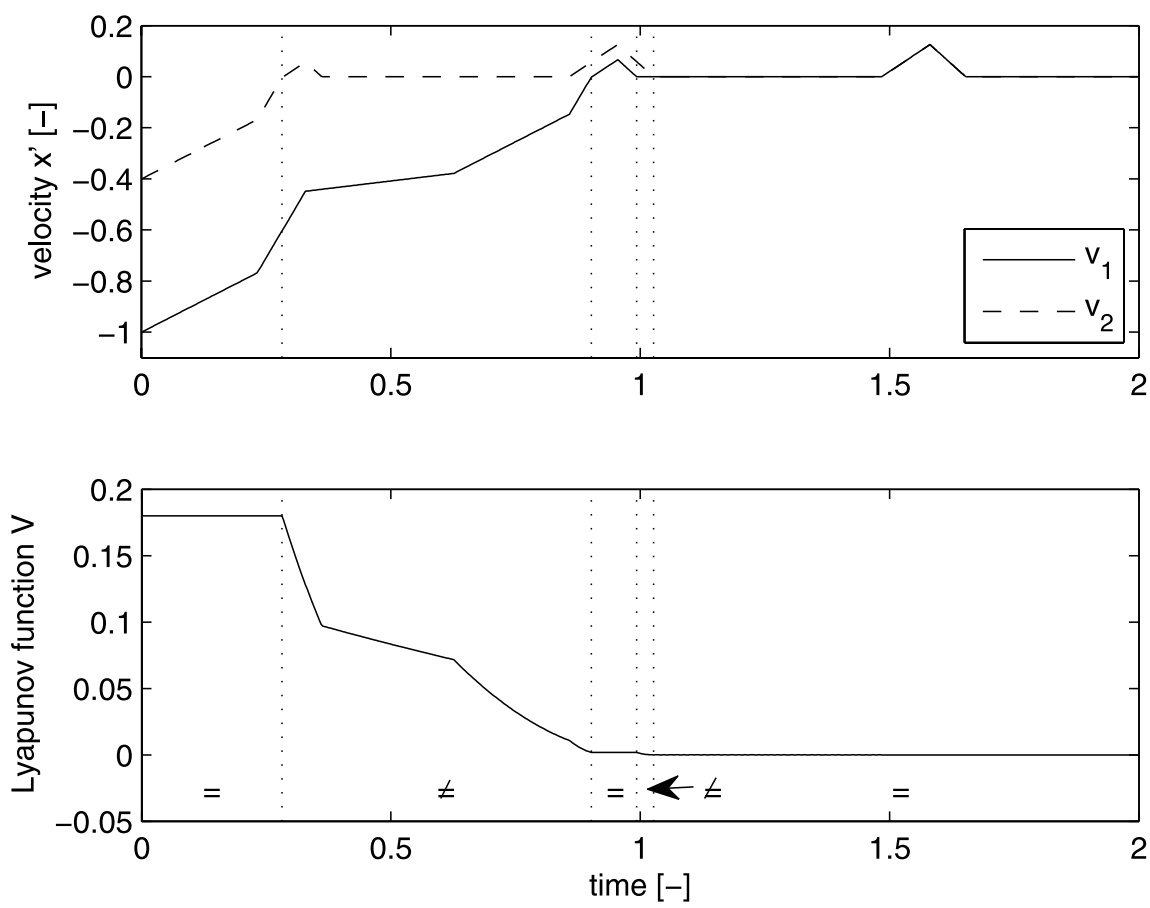

Summarizing, although the time derivative of the Lyapunov function is only negative semi-definite, it has been shown that in the time intervals in which $V^{\prime}=0$ the solutions evolve in such a way that always a time interval in which $V^{\prime}<0$ will be reached. This proves that the two solutions $v_{1}$ and $v_{2}$ will converge to each other. Therefore, the steady-state solution in terms of velocity is unique and is independent of the initial velocity of the mass. In other words, depending on the system parameters, different types of steadystate motion occur as discussed in Sect. 5.1. However, each steady-state solution is unique. There are no coexisting steady-state solutions.

\section{Positioning accuracy in the stopping phase}

For stopping and accurate positioning of the mass, an increased friction coefficient $\mu_{2}$, as described earlier in Sect. 3.3, is used. Depending on the weight distribution of the mass over the two table regions with two different Coulomb friction coefficients $\mu_{1}$ and $\mu_{2}$, the effective friction coefficient $\bar{\mu}$ experienced by the mass can be calculated using (9). Due to the existence of a low friction and a high friction region, the system dynamics in the friction transition region, see (5) and (9), is given by:

$$
-\left(1+\frac{c_{\mu}-1}{w}\left(x-x_{\mu}\right)\right) \operatorname{Sign}\left(x^{\prime}\right) \ni x^{\prime \prime}+y^{\prime \prime}
$$

As long as $y_{i}^{\prime \prime}<1+\left(\left(c_{\mu}-1\right)\left(x-x_{\mu}\right) / w\right)$ for $i=$ 2,3 , the mass will be able to stick in the high friction region.

\subsection{Definition of positioning accuracy}

Before defining and studying the accuracy of the end position of the mass, first a discussion on the derivation of the end position itself will be given. Consider the situation sketches in Figs. 14 and 15. It is assumed that the mass will enter the high friction region in a steady-state motion. The end position of the mass will depend on the state of the mass $\left[x, x^{\prime}\right]^{T}$ (corresponding to a certain phase of the periodic table motion) when it reaches $x=x_{\mu}$ (see Fig. 2) for the first time. This state will obviously depend on the initial state $\left[x_{0 s}, x_{0 s}^{\prime}\right]^{T}$ (the subscript ' $s$ ' stresses the fact that the initial conditions are chosen corresponding to a steady-state solution) at $t=t_{0}$, the phase of the periodic excitation signal at $t=t_{0}$, and on $x_{\mu}-x_{0 s}$. 


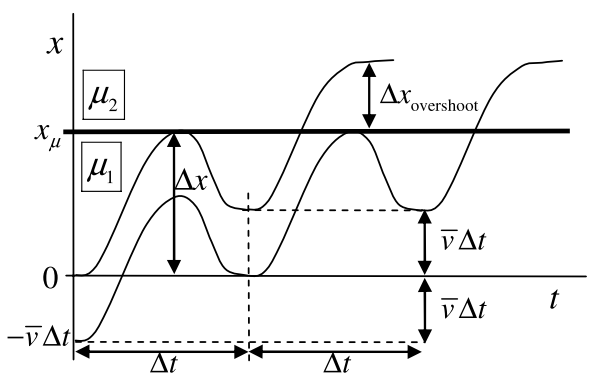

Fig. 14 Situation sketch of when the mass is stopped

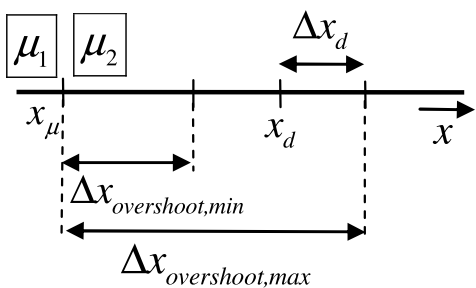

Fig. 15 Region of end positions where the mass can stop

The position where the mass stops, is defined by

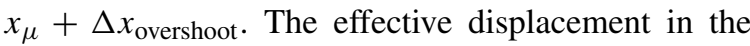
low friction region, during one excitation period in steady state, can be written as $\bar{v} \Delta t$ ( $\bar{v}$ is the average velocity during one excitation period). The maximum displacement in one period is defined as $\Delta x$. To include all possible ways of entering the high friction region in steady state, define $x_{\mu}=\Delta x$ and vary the initial positions corresponding to steady-state $x_{0 s}$ in the range $-\bar{v} \Delta t \leq x_{0 s} \leq 0$. Corresponding initial velocities $x_{0 s}^{\prime}$ are used to assure steady-state motion from the start. Now, collecting all overshoots $\Delta x_{\text {overshoot }} \in$ $\left[\Delta x_{\text {overshoot, } \min ,}, \Delta x_{\text {overshoot, } \max }\right]$ for all initial conditions corresponding to steady-state responses, will provide a range of the possible end positions of the mass. The positioning error is now defined as:

$\Delta x_{d}=\frac{\Delta x_{\text {overshoot, } \max }-\Delta x_{\text {overshoot }, \text { min }}}{2}$

The desired end position can now be defined as $x_{d}=x_{\mu}+\Delta x_{\text {overshoot, } \min }+\Delta x_{d}$, see Fig. 15 .

\subsection{Results}

The positioning accuracy will, next to system properties, obviously depend on the prescribed table motion. In Sect. 5, the stroke and frequency of the table were fixed and the accelerations $y_{2}^{\prime \prime}$ and $y_{3}^{\prime \prime}$ were varied. For different combinations of $y_{2}^{\prime \prime}$ and $y_{3}^{\prime \prime}$, the positioning error is calculated and stored. In this section and in Sect. 7.3, the stroke and the excitation period of the table are set to respectively $\Delta y=0.084$ and $\Delta t=1$. The dimensionless width is chosen to be $w=0.05$. It is assumed that $c_{\mu}=2.5$ such that the friction coefficient in the high friction region is $\mu_{2}=2.5 \mu$. Hence, the interesting region of accelerations is limited to $y_{i}^{\prime \prime}<1+\left(c_{\mu}-1\right)\left(x-x_{\mu}\right) / w$, so to accelerations $y_{i}^{\prime \prime}<2.5$ (enabling the mass to permanently stick to the table). Remarkably, as will be shown below, a design space for $y_{2}^{\prime \prime}$ and $y_{3}^{\prime \prime}$ can be identified where the positioning error is $\Delta x_{d}=0$.

Consider the following two table acceleration settings: $\left(y_{2}^{\prime \prime}, y_{3}^{\prime \prime}\right)=(2.3,1.3)$ and $\left(y_{2}^{\prime \prime}, y_{3}^{\prime \prime}\right)=(1.8,1.1)$. In Fig. 16, the two corresponding phase portraits are shown. For each setting, two responses corresponding to two different initial conditions are shown. The two dashed vertical lines respectively indicate the positions, where the high friction region is starting $\left(x_{\mu}\right)$, and from where it is possible for the mass to permanently stick to the table $\left(x_{\text {stick, min }}\right)$. The position on the table, from where the mass is able to permanently stick to the table, is determined by the following condition:

$\max \left(y_{i}^{\prime \prime}\right)=1+\frac{c_{\mu}-1}{w}\left(x-x_{\mu}\right)$

Using this condition, the position $x_{\text {stick, min }}$ can be calculated to be:

$x_{\text {stick, } \min }=x_{\mu}+w \frac{\max \left(y_{i}^{\prime \prime}\right)-1}{c_{\mu}-1}$

In the lower plot of Fig. 16, the end positions are clearly different. In the upper plot of Fig. 16, however, the mass stops at the same position for both trajectories, although the initial conditions are different: an accumulation position can be identified. A detailed analysis of this accumulation position will be presented in Sect. 7.3. There, among others, it will be shown that depending on the parameter values, sometimes for all initial conditions but also sometimes for only a part of the initial conditions, accumulation results. When accumulation occurs, the same type of trajectory repeats itself on smaller and smaller scales up to the accumulation position. Note that the accumulation position in theory will only be reached for $t \rightarrow \infty$. Interestingly, when accumulation occurs, it will be shown that the trajectories accumulate to the 
Fig. 16 Phase portraits of two different settings when stopping the mass
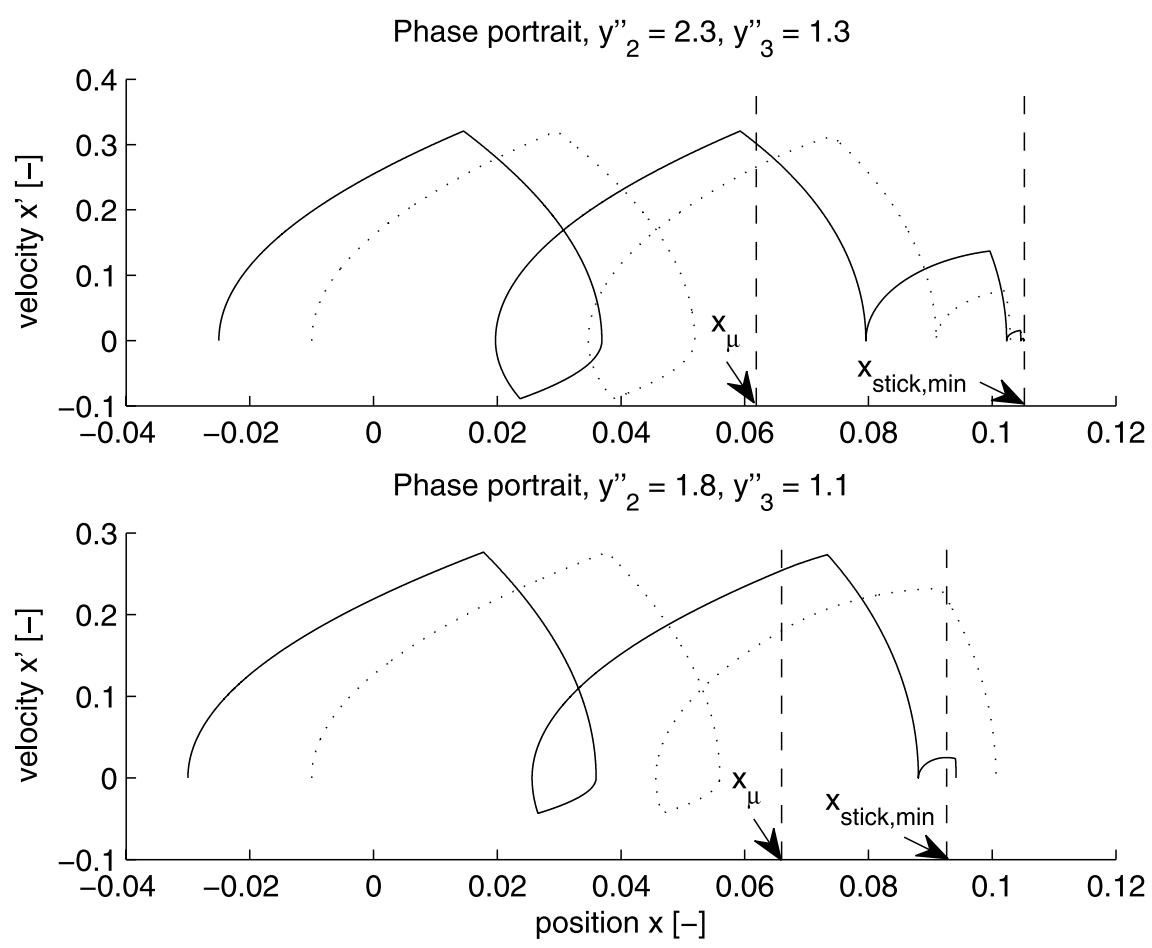

position from where it is possible for the mass to permanently stick, i.e. $x_{\text {stick, min }}$. Figure 17 combines several analysis results showing a surface plot (A) of the objective function value $f$, see (11), a case-plot (B) indicating the type of motion, an accumulation identification plot (C), and a surface plot with the positioning accuracy (D). From the function value plot (A) it is clear that a high value for $y_{2}^{\prime \prime}$ results in fast movement of the mass, but $y_{2}^{\prime \prime}$ should be sufficiently low to allow sticking in the high friction region. Simultaneously, a low value for $y_{3}^{\prime \prime}$ increases the chance of a case 1 type of motion, see plot (B). Fortunately, this is also the parameter space in which the lowest positioning error can be achieved, see plot (D). When plot (C) is compared to the positioning error plot (D), it becomes clear that, for the current parameter settings and used initial conditions, the occurrence of accumulation results in extreme positioning accuracy of the mass $\left(\Delta x_{d}=0\right)$.

\subsection{Analysis of the accumulation position}

The previous section showed that for some parameter settings, the mass stops at a unique end position, even though the initial conditions are different, which leads to ultimate positioning accuracy of the mass. It is now assumed that the mass enters the high friction region with a case 1 steady-state motion without any negative relative velocity. This corresponds to the lowest values of $f$ in the region with low acceleration values, see subplot (A) in Fig. 17. This case 1 motion guarantees that the mass will stay in the high friction region once it entered it.

In this section, analytic expressions will be derived for the subsequent positions where relative velocities $x^{\prime}=0$ are found in the region $x>x_{\mu}$. Simulations based on these expressions indicate that, in case of accumulation, the distance between these positions will become shorter and shorter and that accumulation to a fixed position will occur.

In the transition region $\left(x_{\mu} \leq x \leq x_{\mu}+w\right)$, when the mass is moving, so in forward slip, the equation of motion (38) can be written as:

$x^{\prime \prime}(t)+b_{1} x(t)=b_{3}(t)$

where $b_{1}=\left(c_{\mu}-1\right) / w, b_{3}(t)=b_{2}-y^{\prime \prime}(t)$, and $b_{2}=$ $\left(\left(c_{\mu}-1\right) x_{\mu} / w\right)-1$. Thus, in essence, the system behaves as a forced mass-spring system in the friction transition region. The general analytic solution to dif- 
Fig. 17 Objective function value $f(\mathbf{A})$, case-plot $(\mathbf{B})$, accumulation identification plot $(\mathbf{C})$, positioning error $\Delta x_{d}(\mathbf{D})$

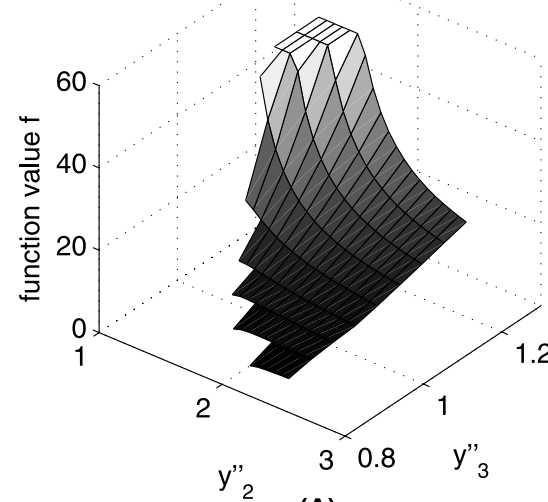

(A)

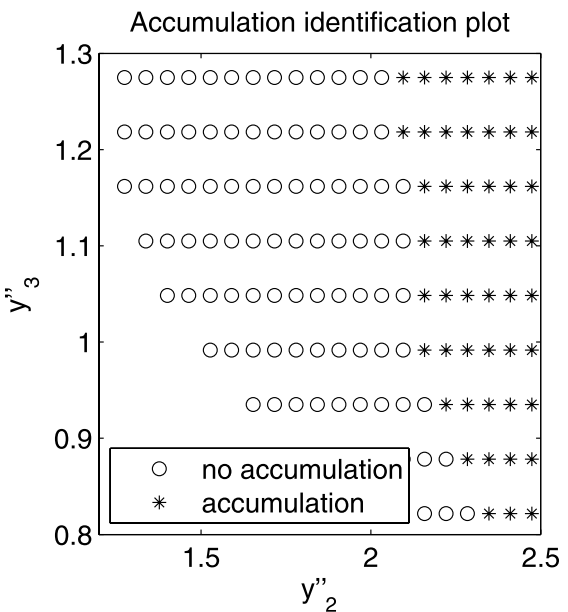

(C)

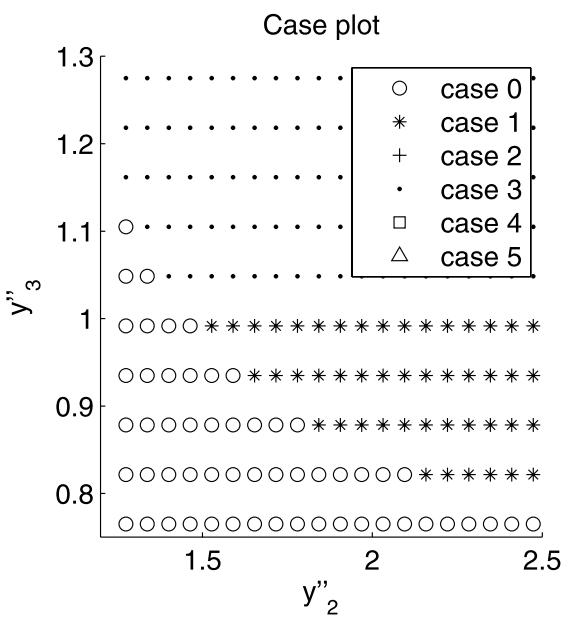

(B)

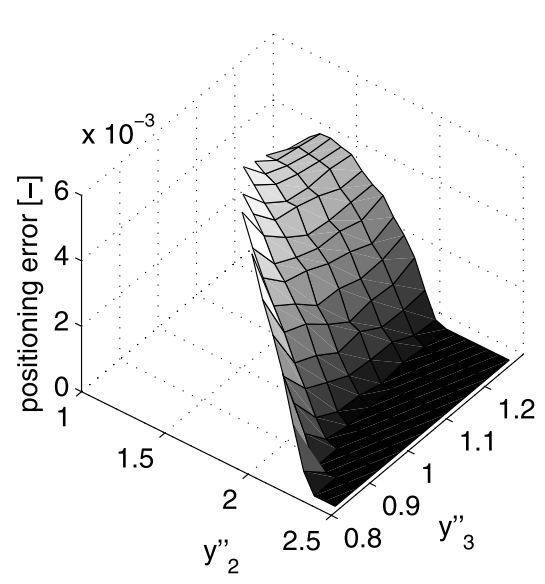

(D) ferential equation (42) can be written as follows:

$$
\begin{aligned}
x(t)= & \frac{b_{3}}{b_{1}}+d_{1} \cos \left(\sqrt{b_{1}}\left(t-t^{*}\right)\right) \\
& +d_{2} \sin \left(\sqrt{b_{1}}\left(t-t^{*}\right)\right), \\
x^{\prime}(t)= & -d_{1} \sqrt{b_{1}} \sin \left(\sqrt{b_{1}}\left(t-t^{*}\right)\right) \\
& +d_{2} \sqrt{b_{1}} \cos \left(\sqrt{b_{1}}\left(t-t^{*}\right)\right)
\end{aligned}
$$

where the constants $d_{1}$ and $d_{2}$ are determined by the conditions at the time $t=t^{*}$, at which the mass enters the transition region, i.e. $x\left(t^{*}\right)=x_{\mu}$ :

$d_{1}=x\left(t^{*}\right)-\frac{b_{3}}{b_{1}}$,

$$
d_{2}=\frac{x^{\prime}\left(t^{*}\right)}{\sqrt{b_{1}}}
$$

The time at which the velocity reaches zero for the first time for $t>t^{*}$ can be calculated analytically:

$$
t_{x^{\prime}=0}=t^{*}+\frac{1}{\sqrt{b_{1}}} \arcsin \left(\frac{d_{2}}{\sqrt{d_{1}^{2}+d_{2}^{2}}}\right)
$$

Now, our goal is to find expressions for the subsequent positions where the mass sticks to the table. By studying these subsequent stick positions, or actually the mapping between them, it is possible to explain the accumulation to a unique end position. The position at which stick occurs for the $n$th time, is denoted by $x_{n}$. For $n=1, x_{1}$ can be calculated by substituting (47) into (43). To make the notation more compact, a new 
time scale $\tau$ is introduced, which is zero at the moment when the mass gets out of the first stick phase in the transition region. The mass will stick at the $y_{3}^{\prime \prime}$ part of the trajectory. The mass therefore starts to slip at $\tau=0$ when the acceleration changes from $y_{1}^{\prime \prime}=0$ to $-y_{2}^{\prime \prime}$. The following values apply at $\tau=0$ :

$b_{3,1}=b_{2}+y_{2}^{\prime \prime}$,

$d_{1,1}=x_{n}-\frac{b_{3,1}}{b_{1}}$,

$d_{2,1}=\frac{0}{\sqrt{b_{1}}}=0$

Note that $b_{1}$ and $b_{2}$ are constant in the transition region. The first part of the analytic solution for $0 \leq \tau \leq$ $t_{2}-t_{1}$ can therefore be written as follows:

$x(\tau)=\frac{b_{3,1}}{b_{1}}+d_{1,1} \cos \left(\sqrt{b_{1}} \tau\right)$,

$x^{\prime}(\tau)=-d_{1,1} \sqrt{b_{1}} \sin \left(\sqrt{b_{1}} \tau\right)$

At $\tau=t_{2}-t_{1}$, the acceleration changes from $-y_{2}^{\prime \prime}$ to $y_{3}^{\prime \prime}$. At $\tau=t_{2}-t_{1}$, the following values apply:

$b_{3,2}=b_{2}-y_{3}^{\prime \prime}$,

$d_{1,2}=x\left(t_{2}-t_{1}\right)-\frac{b_{3,1}}{b_{1}}$,

$d_{2,2}=\frac{x^{\prime}\left(t_{2}-t_{1}\right)}{\sqrt{b_{1}}}$

which yields the following analytic solution for time interval $t_{2}-t_{1} \leq \tau \leq \tau_{x^{\prime}=0}$, where $\tau_{x^{\prime}=0}$ is the time at which the relative velocity $x^{\prime}$ becomes zero again:

$$
\begin{aligned}
x(\tau)= & \frac{b_{3,2}}{b_{1}}+d_{1,2} \cos \left(\sqrt{b_{1}}\left(\tau-\left(t_{2}-t_{1}\right)\right)\right) \\
& +d_{2,2} \sin \left(\sqrt{b_{1}}\left(\tau-\left(t_{2}-t_{1}\right)\right)\right) \\
x^{\prime}(\tau)= & -d_{1,2} \sqrt{b_{1}} \sin \left(\sqrt{b_{1}}\left(\tau-\left(t_{2}-t_{1}\right)\right)\right) \\
& +d_{2,2} \sqrt{b_{1}} \cos \left(\sqrt{b_{1}}\left(\tau-\left(t_{2}-t_{1}\right)\right)\right)
\end{aligned}
$$

Using (47), the mass gets into stick in the transition region for the second time, at time:

$$
\tau_{x^{\prime}=0}=\left(t_{2}-t_{1}\right)+\frac{1}{\sqrt{b_{1}}} \arcsin \left(\frac{d_{2,2}}{\sqrt{d_{1,2}^{2}+d_{2,2}^{2}}}\right)
$$

The displacement at $\tau_{x^{\prime}=0}$ will be denoted by $x_{n+1}=x\left(\tau_{x^{\prime}=0}\right)$. After algebraic manipulation the following mapping between subsequent positions $x_{n}$ and $x_{n+1}$ can be found:

$$
\begin{aligned}
x_{n+1}= & \frac{b_{2}-y_{3}^{\prime \prime}}{b_{1}} \\
& +\left\{\left(\frac{y_{2}^{\prime \prime}+y_{3}^{\prime \prime}}{b_{1}}+\left(x_{n}-\frac{b_{2}+y_{2}^{\prime \prime}}{b_{1}}\right)\right.\right. \\
& \left.\times \cos \left(\sqrt{b_{1}}\left(t_{2}-t_{1}\right)\right)\right)^{2} \\
& \left.+\left(\left(x_{n}-\frac{b_{2}+y_{2}^{\prime \prime}}{b_{1}}\right) \sin \left(\sqrt{b_{1}}\left(t_{2}-t_{1}\right)\right)\right)^{2}\right\}^{\frac{1}{2}}
\end{aligned}
$$

The fixed point of this mapping can be calculated:

$x_{n}=x_{n+1}=\frac{b_{2}+y_{2}^{\prime \prime}}{b_{1}}=x_{\text {stick }, \min }$

Recall that $b_{1}=\left(c_{\mu}-1\right) / w$ and $b_{2}=\left(c_{\mu}-1\right) x_{\mu} /$ $w-1$, so that in fact the fixed point of the mapping is the position $x_{\text {stick, min }}$, from where permanent stick is possible, see (41). In Fig. 18, mapping (59) is shown for settings $y_{2}^{\prime \prime}=2.2, y_{3}^{\prime \prime}=0.9, \Delta t=1.05$, and $\Delta y=0.084$. Note that this also specifies $t_{2}-t_{1}$ appearing in (59), see Appendix. The left and the right dashed vertical lines in Fig. 18 indicate respectively the starting position of the $\mu_{2}$ friction region $\left(x=x_{\mu}\right)$, and from where the mass is able to permanently stick $\left(x=x_{\text {stick, min }}\right)$. Indeed, the fixed point of the mapping is located at the same position as $x_{\text {stick, min }}$.

Note again that in fact the end position, in case of accumulation to this fixed point, is reached for $t \rightarrow \infty$. In a practical situation, however, the end position will be reached in finite time. Consider a dimensionless scalar $p$, where $0 \leq p \leq 1$. For the cases where accumulation occurs in Fig. 17, the position $x_{\mu}+p\left(x_{\text {stick, } \min }-x_{\mu}\right)$ is reached in at most 5 period times for $p=0.99$, and in at most 7 period times for $p=0.999$. This has been verified by time-stepping simulations.

The initial conditions that determine the first stick position $x_{1}$, can influence the occurrence of accumulation. Consider again Fig. 18, which by means of the middle dashed vertical line shows that, depending on the initial conditions, the mass can stick to the table 
Fig. 18 Depending on the initial conditions, accumulation to a fixed end position is possible

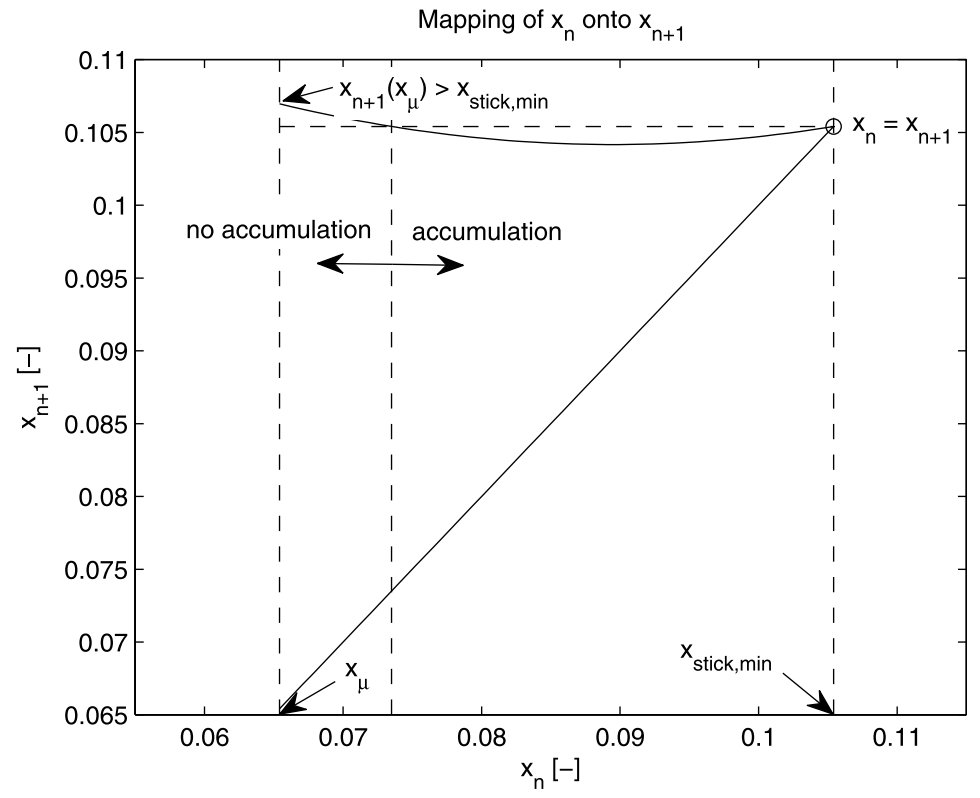

at a position $x>x_{\text {stick, min }}$, or can exhibit the accumulating behavior and end up at $x=x_{\text {stick, min }}$. Note that accumulation to an end position will always occur if $x_{n+1}\left(x_{\mu}\right)<x_{\text {stick, min }}$, and is then independent of the initial conditions.

\section{Conclusions and recommendations}

In this paper, one-dimensional self-alignment of a mass via stick-slip vibrations has been studied. Using a suitable periodic table trajectory, it is possible to move the mass into a desired direction and using an increase in the friction coefficient it is possible to stop the mass at a certain end position.

The first objective of this paper has been to design a trajectory for the table, such that the mass moves to a desired end position in the least possible amount of time, given some table motion constraints. The design of a table motion profile, consisting of three time intervals with different constant accelerations has been discussed. For the mass, analytic steady-state solutions have been derived, and a classification of the types of steady-state responses of the mass has been given.

Using Floquet theory for discontinuous systems, the local stability of the solutions has been proven. Due to the freedom in position, one Floquet multiplier will always be equal to one. Furthermore, using a Lyapunov analysis, convergence of the velocity response has been proven. In other words, different velocity solutions will converge to each other, independent of the initial velocities. This convergence implies uniqueness of the velocity response of the mass.

The second objective of this paper has been to stop the mass at a desired end position with the best possible positioning accuracy. For certain parameter settings, an interesting phenomenon is observed in the transition region from low friction to high friction, namely accumulation of the mass position to a unique end position. This accumulation behavior benefits the positioning accuracy in an ultimate manner. In essence, the system behaves as a forced mass-spring system in this region, which allows for an analytical study of the accumulation behavior. Subsequent stickpositions have been related to each other resulting in a discrete mapping. It is shown that the fixed point of this mapping is exactly the position from where permanent stick becomes possible. Under certain conditions, accumulation will always occur, independent of the initial conditions.

Recommendations for future research are: (1) experimental verification of the analyzed behavior; (2) analysis of models with resembling, but more realistic, table motion profiles, i.e. finite values for the jerk should be introduced at times when the table acceleration values change; (3) use of enhanced friction models; (4) extension to two-dimensional models de- 
scribing general planar motion of the mass; and (5) extension to self-alignment of multiple masses.

Open Access This article is distributed under the terms of the Creative Commons Attribution Noncommercial License which permits any noncommercial use, distribution, and reproduction in any medium, provided the original author(s) and source are credited.

\section{Appendix: Details of the prescribed table motion generation using three acceleration parts}

In order to calculate a table trajectory that satisfies the 7 constraints formulated in Sect. 4.4.3, it is necessary to find explicit expressions for the following 5 variables: $t_{1}, t_{2}, t_{3}, y_{\text {ini }}$, and $y_{\text {ini }}^{\prime}$. Note that the following two variables are already explicitly known: $y_{1}^{\prime \prime}=0$ and $t_{0}=0$. It is therefore only needed to solve 5 constraint equations, which can be derived from the constraints formulated in Sect. 4.4.3. Using Fig. 7 the following 5 equations can be derived:

1. $y_{\text {ini }}=\frac{1}{2} y_{3}^{\prime \prime}\left(t_{3}-t_{y^{\prime}=0,2}\right)^{2}$

2. $t_{3}-t_{0}=\Delta t$

3. $\frac{1}{2} y_{2}^{\prime \prime}\left(t_{2}-t_{y^{\prime}=0,1}\right)^{2}+\frac{1}{2} y_{3}^{\prime \prime}\left(t_{y^{\prime}=0,2}-t_{2}\right)^{2}=\Delta y$

4.

$$
\begin{aligned}
y_{\text {ini }}^{\prime}\left(t_{1}-t_{0}\right)+\frac{1}{2} y_{2}^{\prime \prime}\left(t_{y^{\prime}=0,1}-t_{1}\right)^{2} & \\
& +\frac{1}{2} y_{3}^{\prime \prime}\left(t_{3}-t_{y^{\prime}=0,2}\right)^{2} \\
= & \frac{1}{2} y_{2}^{\prime \prime}\left(t_{2}-t_{y^{\prime}=0,1}\right)^{2}+\frac{1}{2} y_{3}^{\prime \prime}\left(t_{y^{\prime}=0,2}-t_{2}\right)^{2}
\end{aligned}
$$

5. $y_{3}^{\prime \prime}\left(t_{3}-t_{2}\right)=y_{2}^{\prime \prime}\left(t_{2}-t_{1}\right)$

For convenience, in these equations two additional unknowns are introduced, namely $t_{y^{\prime}}=0,1$ and $t_{y^{\prime}=0,2}$, which are the times at which the velocity $y^{\prime}$ of the table becomes zero. Therefore, to solve the set of equations, two additional equations are needed, which define these two additional unknowns:

6. $y_{\text {ini }}^{\prime}-y_{2}^{\prime \prime}\left(t_{y^{\prime}=0,1}-t_{1}\right)=0$

7. $y_{\text {ini }}^{\prime}-y_{3}^{\prime \prime}\left(t_{3}-t_{y^{\prime}=0,2}\right)=0$

Now 7 equations are available in the 7 unknowns $t_{1}, t_{2}, t_{3}, y_{\text {ini }}, y_{\text {ini }}^{\prime}, t_{y^{\prime}=0,1}$, and $t_{y^{\prime}=0,2}$. This set of equations is solved by the symbolic toolbox of Matlab. The explicit solutions are not given here because the expressions are too long to display.

Please note that actually there is a second case that needs to be considered. If the initial velocity $y_{\text {ini }}^{\prime}$ appears to be smaller than 0 , then the previously discussed mathematical description of the constraints is not valid anymore. However, a completely equivalent reasoning as for the case $y_{\text {ini }}^{\prime}>0$ can be followed. Therefore, the second case is not discussed in detail here. Moreover, note that it is possible that no physical solutions exist for the above specified equations if, for example, a large displacement $\Delta y$ is demanded in a short amount of time $\Delta t$, using small acceleration values $y_{2}^{\prime \prime}$ and $y_{3}^{\prime \prime}$. In that case the solutions of some variables will be complex valued, lacking a physical interpretation.

Acknowledgements This work was supported in part by a grant from Miami University's School of Engineering and Applied Sciences for International collaborations.

\section{References}

1. Armstrong-Hélouvry, B., Dupont, P., Canudas De Wit, C.: A survey of models, analysis tools and compensation methods for the control of machines with friction. Automatica 30(7), 1083-1138 (1994)

2. Berger, E.J.: Friction modeling for dynamic system simulation. Appl. Mech. Rev. 55(6), 535-577 (2002)

3. Blekhman, I.I.: Vibrational Mechanics-Nonlinear Dynamic Effects, General Approach, Applications. World Scientific, Singapore (2000)

4. Chernous'ko, F.L.: Analysis and optimization of the motion of a body controlled by means of a movable internal mass. PMM J. Appl. Math. Mech. 70, 819-842 (2006)

5. Dankowicz, H., Normark, A.B.: On the origin and bifurcations of stick-slip oscillations. Physica D 136, 280-302 (2000)

6. Den Hartog, J.P.: Forced vibrations with combined Coulomb and viscous damping. ASME J. Appl. Mech. 53, 107-115 (1930)

7. Duarte, F.B., Machado, J.T.: Fractional describing function of systems with Coulomb friction. Nonlinear Dyn. 56, 381387 (2009)

8. Fidlin, A., Thomsen, J.J.: Predicting vibration-induced displacement for a resonant friction slider. Eur. J. Mech. Solids 20, 807-831 (2001)

9. Frutiger, D.R., Vollmers, K., Kratochvil, B., Nelson, B.J.: Small, fast, and under control: wireless resonant magnetic micro-agents. Exp. Robot. 54, 169-178 (2009)

10. Galvanetto, U., Bishop, S.R.: Dynamics of a simple damped oscillator undergoing stick-slip vibrations. Meccanica 34, 337-347 (1999)

11. Leine, R.I., Nijmeijer, H.: Dynamics and Bifurcations of Non-Smooth Mechanical Systems. LNACM, vol. 18. Springer, Berlin (2004) 
12. Leine, R.I., van de Wouw, N.: Stability and Convergence of Mechanical Systems with Unilateral Constraints. LNACM, vol. 36. Springer, Berlin, Heidelberg, New-York (2008)

13. Luo, A.C.J., Gegg, B.C.: Stick and non-stick periodic motions in periodically forced oscillators with dry friction. J. Sound Vib. 291, 132-168 (2006)

14. Moreau, J.J.: Unilateral contacts and dry friction in finite freedom dynamics. Non-Smooth Mech. Appl., 302 (1988)

15. Natsiavas, S.: Stability of piecewise linear oscillators with viscous and dry friction damping. J. Sound Vib. 217(3), 507-522 (1998)

16. Pogromsky, A.Yu., Heemels, W.P.M.H., Nijmeijer, H.: On solution concepts and well-posedness of linear relay systems. Automatica 39, 2139-2147 (2003)

17. Rakotondrabe, M., Haddab, Y., Lutz, P.: Development, modeling, and control of a micro-/nanopositioning 2-dof stick-slip device. IEEE/ASME Trans. Mechatron. 14(6), 733-745 (2009)

18. Reznik, D.S., Canny, J.F., Alldrin, N.: Leaving on a plane jet. In: IEEE International Conference on Intelligent Robots and Systems, Maui, HI, pp. 202-207, October (2001)
19. Sato, K., Ito, K., Hata, S., Shimokohbe, A.: Self-alignment of microparts using liquid surface tension-behavior of micropart and alignment characteristics. Precis. Eng. 27, 42$50(2003)$

20. Shaw, S.W.: On the dynamical response of a system with dry friction. J. Sound Vib. 108(2), 302-325 (1986)

21. Stein, G.J., Zahoranskỳ, R.: On dry friction modelling and simulation in kinematically excited oscillatory systems. J. Sound Vib. 311, 74-96 (2008)

22. Studer, C.: Numerics of Unilateral Contacts and FrictionModeling and Numerical Time Integration in Non-Smooth Dynamics. LNACM, vol. 47. Springer, Berlin, Heidelberg (2009)

23. Thomsen, J.J.: Some general effects of strong highfrequency excitation: stiffening, biasing, and smoothening. J. Sound Vib. 253, 807-831 (2002)

24. Thomsen, J.J., Fidlin, A.: Analytical approximations for stick-slip vibration amplitudes. Int. J. Non-Linear Mech. 38, 389-403 (2003) 